\title{
The structure of optimal consumption streams in general incomplete markets
}

\section{Journal Article}

\section{Author(s):}

Malamud, Semyon; Trubowitz, Eugene

Publication date:

2007

Permanent link:

https://doi.org/10.3929/ethz-b-000004713

Rights / license:

In Copyright - Non-Commercial Use Permitted

Originally published in:

Mathematics and Financial Economics 1(2), https://doi.org/10.1007/s11579-007-0006-y 


\title{
The structure of optimal consumption streams in general incomplete markets
}

\author{
Semyon Malamud · Eugene Trubowitz
}

Received: 15 May 2007 / Accepted: 20 September 2007 / Published online: 1 November 2007

C Springer-Verlag 2007

\begin{abstract}
We prove that for any incomplete market and any concave utility function the marginal propensities to consume and to save are always positive. Furthermore, we introduce a class of incomplete markets that includes almost all well known examples of market incompleteness in finance and macroeconomics. Two concrete examples are idiosyncratic income shocks and general, diffusion driven incompleteness. For all markets in our class we explicitly solve the associated utility maximization problem by a recursive construction and derive many important properties. For example, precautionary savings and the diminishing marginal propensity to consume. Effectively, the class is characterized by these two economic properties. We also prove that the growth rate of consumption is always larger when markets are incomplete and that precautionary savings are monotone increasing in the size of idiosyncratic risk. Our construction can be implemented computationally by an efficient, robust numerical scheme.
\end{abstract}

Keywords Optimal consumption stream · Incomplete markets · Idiosyncratic risk · Convexity $\cdot$ Marginal propensity to consume

JEL Classification $\mathrm{G} 11 \cdot \mathrm{E} 21 \cdot \mathrm{D} 91 \cdot \mathrm{D} 81$

\section{Introduction}

The optimal consumption of a rational economic agent is a function of many economic factors such as asset prices, income and wealth. One of the most important problems in economics is to understand the structure of this consumption function.

We thank two anonymous referees for useful comments and remarks.

S. Malamud $(\varangle) \cdot$ E. Trubowitz

ETHZ, D-MATH, Rämistrasse 101, 8092 Zurich, Switzerland

e-mail: semka@math.ethz.ch

E. Trubowitz

e-mail: trub@math.ethz.ch 
There is a large literature, analyzing the "empirical" consumption function using empirical data, see, e.g., [22]. There is also a large literature devoted to theoretical analysis of the optimal consumption of rational, utility maximizing agents. The standard textbook on this literature is [12].

When markets are complete, the structure of the consumption function becomes very simple and transparent. Optimal consumption does not depend on the whole structure of the income stream, but only on its intertemporal value, called the intertemporal wealth. In particular, for the scale invariant CRRA utility functions, optimal consumption is a linear function of wealth.

When markets are incomplete, the behavior changes dramatically and consumption becomes a highly non linear function of both wealth and the whole income stream. Except for the rather well understood case of standard, macroeconomic incomplete markets with only one period risk free bonds (see, e.g., [21]), almost nothing is known about the structure of the consumption function in general incomplete markets in the presence of risky assets. In this paper we address the following basic economic questions:

(1) Are the marginal propensities to consume and to save positive for general incomplete markets? That is, we ask whether consumption and savings are monotone increasing in wealth.

(2) Do wealthy people consume a smaller fraction of their wealth than poor people? Equivalently, we ask whether marginal propensity to consume is diminishing, i.e., consumption is a concave function of wealth.

(3) How does consumption depend on uninsurable income risk? In particular, does future income uncertainty force an agent to save more, i.e., does it generate precautionary savings?

Of course, to have any hope of answering these three questions, we must "get our hands on" the consumption function in general incomplete markets. That is, construct it in some "explicit form". The first obstacle to an explicit construction is that the budget constraints are expressed in terms of an infinite set of state price densities. One of the key ideas of our construction is to rewrite the budget constraints and first order conditions in terms of the orthogonal projections on the so-called payoff subspaces and a special, unique, aggregate state price density process (Proposition 2.9). This allows us to explicitly construct the optimal consumption stream for arbitrary incomplete markets by a recursive procedure backwards in time (Theorem 2.14) from the final horizon to the initial time period. In particular, our explicit recursive structure immediately implies that both marginal propensities to consume and to save are positive. Interestingly enough, even though current consumption is monotone increasing in current wealth, future consumption is not necessarily monotone in current wealth. This phenomenon reflects the fact that there are nonlocal maps in our recursive construction. We have discovered a large class of incomplete markets for which these maps are local.

Our new class $\mathfrak{C}$ of incomplete markets is characterized by natural positivity properties. For this class, the recursive procedure reduces to inverting functions of one variable. It is an important class because it includes almost all well known examples of market incompleteness in finance and macroeconomics. Three concrete examples are idiosyncratic income shocks, discrete time diffusion driven incompleteness and markets with only one period risk free bonds. Our solution procedure covers both traditional, time and state separable utilities, as well as, the recursive utilities of Epstein-Zin type.

The incomplete markets in our class $\mathfrak{C}$ have the following important properties: 
- They are directly characterized by the presence of a hedgeable filtration, contained in the underlying filtration. The states in the hedgeable filtration are precisely those that can be perfectly hedged against by trading.

- They are economically characterized by the properties of diminishing marginal propensity to consume and the presence of precautionary savings. Furthermore, only for these incomplete markets future consumption is monotone increasing in current wealth.

- One period risk free bonds are the only assets available for trade in standard, macroeconomic models (see, e.g., [21]). For this reason, the Euler equations have a particularly simple form: the marginal utility process is a martingale (see [21, Chap. 16]). For genuinely risky assets, the Euler equations are far more complicated. In our class $\mathfrak{C}$, this difficulty is surmounted by expressing the Euler equations in terms of the hedgeable filtration and the aggregate state price density process. Namely, the quotient of the marginal utility and the aggregate state price density process is a martingale. This device enables us to generalize most of the standard, macroeconomic analysis (see, e.g., [21]) to the case of risky assets.

- The equations defining equilibria for incomplete markets in $\mathfrak{C}$ can be written in a relatively simple form purely in terms of the unique, positive, aggregate state price density process, analogous to Arrow-Debreu equilibria for complete markets. We have developed new methods for analyzing them, see, e.g., [23].

- The incompleteness captured in $\mathfrak{C}$ is ideally suited for market consistent pricing of insurance products, see, e.g., [24].

The economic intuition underlying our class is simple. It is particularly transparent when pictured in terms of caricature, "smeared out", one period Arrow state contingent securities.

A "sharp", one period Arrow security pays one dollar in a particular, given state at the next period. A "smeared out", one period Arrow security pays one dollar on a set of states at the next period. Trading in a small number of fundamental, multiperiod securities in our incomplete markets generates a basis set of smeared out, one period Arrow securities, in analogy with dynamically complete markets (see, e.g., [7]).

For example, if idiosyncratic income risk is the only source of incompleteness, there are aggregate events that determine the state of the financial market that consist of many idiosyncratic events. That is, the aggregate event is smeared out over a collection of idiosyncratic events. Consequently, the true Arrow security corresponding to an aggregate event is "smeared out" over idiosyncratic events.

To make this picture useful, we must get our hands on the smeared out Arrow securities generated by trading in fundamental, multiperiod assets. To do this, we introduce a new mathematical/economic tool that catches them. Namely, the hedgeable filtration.

Let $\mathscr{G}=\left(\mathscr{G}_{t}\right)$ be the underlying filtration (information structure). An incomplete market belongs to our class $\mathfrak{C}$ if and only if there exists a hedgeable subfiltration $\mathscr{H}=\left(\mathscr{H}_{t}\right)$ of $\mathscr{G}$ with the property that any smeared out, one period Arrow security corresponding to an event in $\mathscr{H}_{t+1}$ can be replicated by purchasing a portfolio of assets at time $t$. We note that $\mathscr{G}_{t+1} \supset \mathscr{H}_{t+1} \supset \mathscr{G}_{t}$ whenever there is always a one period risk free bond available for trading. Surprisingly, the characterization of markets in our class at the quasi local level of smeared out Arrow securities is equivalent to the imposition of global economic properties, such as precautionary savings and diminishing marginal propensity to consume. Hedgeable filtrations can be explicitly constructed in most important applications (see Sect. 4).

As we mention above, the recursive structure of the budget constraints and the Euler equations that we have discovered allows us to explicitly construct the optimal consumption stream. Concretely, let $c_{t}$ and $W_{t}$ be the consumption and wealth of an agent at time $t$. Let 
$\mathbf{w}=\left(w_{t}, t=0, \ldots, T\right)$ be his/her income stream. We have explicitly constructed random functions $F_{t}(x, \mathbf{w})$ and $G_{t}(x, \mathbf{w})$ such that, for each $t=T, \ldots, 1$,

$$
\begin{aligned}
W_{t} & =F_{t}\left(c_{t-1}, \mathbf{w}\right) \\
c_{t-1} & =G_{t-1}\left(W_{t-1}, \mathbf{w}\right)
\end{aligned}
$$

A great deal of important economic information about the optimal consumption stream is packed into the random functions $F_{t}$ and $G_{t}$. In particular, both $F_{t}$ and $G_{t}$ are monotone increasing in the first variable for each $t$. One immediate consequence is that, for incomplete markets in the class $\mathfrak{C}$, future consumption is monotone increasing in current wealth. This is not true for general incomplete markets.

The detailed structure of the random functions $F_{t}(W, \mathbf{w})$ and $G_{t}(c, \mathbf{w})$ depends on the underlying utility function $u(c)$. We give a complete description of the class of utility functions $u(c)$ such that, for each $t=0, \ldots, T$, the function $F_{t}$ is jointly concave in the pair $(W, \mathbf{w})$, and $G_{t}$ is jointly convex in $(c, \mathbf{w})$. To our great surprise, the concrete description of this class of utilities depends on the first five derivatives of $u(c)$. Our class contains the HARA utility functions with positive third derivative and, in particular, all CRRA and CARA utilities.

The concavity of $G_{t}(W, \mathbf{w})$ has an important economic significance. By definition, the derivative

$$
\frac{\partial c_{t}}{\partial W_{t}}=\frac{\partial G_{t}\left(W_{t}, \mathbf{w}\right)}{\partial W_{t}}
$$

is the marginal propensity to consume. The concavity of $G_{t}$, with respect to the wealth $W_{t}$, expresses a diminishing marginal propensity to consume.

We now explain the relationship between our work and the existing literature.

Dreze and Modigliani [5] and Carroll and Kimball [2] show that both marginal propensity to consume and marginal propensity to save are positive in the very special case when only one period risk free bonds are available for trading. To the best of our knowledge, nothing is known in the literature about the sign of both propensities in the presence of risky assets. Our Theorem 2.15 establishes this economically important positivity in maximal generality for arbitrary incomplete markets.

Leland [19] and Sandmo [32] were the first to show that a utility function with a positive third derivative (convex marginal utility) is sufficient for precautionary savings (see also [12, Proposition 6.1]). They did this in the special case of a one period model with a single risk free bond (no risky assets). Dreze and Modigliani [5] obtained weaker sufficient conditions (involving the risk free rate), sufficient for precautionary savings with only one period risk free bond. Finally, Menezes and Auten [25] obtained necessary and sufficient conditions for precautionary savings with general one period utility functions and only one period risk free bonds.

In their attempt to include risky assets in their analysis of precautionary savings, Dreze and Modigliani [5, p. 323], write: "Unfortunately, when $r$ is stochastic, this line of reasoning is no longer valid". Here, $r$ is the rate of return on a risky asset. We show that "this line of reasoning" can be made valid if an only if the market is in our class $\mathfrak{C}$. Propositions 5.5 and 5.6 generalize and extend the results of Leland [19] and Sandmo [32] and Dreze and Modigliani [5] in a substantial way, we characterize incomplete markets for which precautionary savings arise.

Miller [28] considered economies with an arbitrary number of periods, but again only with one period risk free bonds and assumed that the risk free rate is constant and independent of time. But, he allowed for an arbitrary idiosyncratic income shocks process. Miller showed 
that for utility functions with positive third derivative, consumption monotonically decreases when the riskiness of labor income shocks increases. Special cases of this general theorem were obtained in [27] and [35]. Note that the market in question is purely idiosyncratically incomplete: the aggregate filtration is trivial, because there is no uncertainty in asset returns. Our Corollary 5.18 can thus be considered as a partial extension of this theorem: consumption is monotone decreasing in the size of idiosyncratic risk for utility functions satisfying the conditions of Theorem 5.15. We do not know whether there is a direct extension of Miller's result to the whole class of idiosyncratically incomplete markets.

Kimball [17] was the first to develop a rigorous theory of precationary savings analogous to the Arrow-Pratt theory of risk aversion. He introduced a new quantity, absolute prudence of a utility function, and showed that it determines the strength of the precautionary savings effect. In particular, he proved that, if the absolute prudence is decreasing(increasing) then labor income uncertainty will raise(lower) the marginal propensity to consume at any given level of consumption. Kimball also considered the effects of portfolio composition (in one period models) on savings. Our techniques allow us to obtain results of this type, but this will be a topic of a separate paper.

The importance of a diminishing marginal propensity to consume was emphasized by Keynes [16, see, e.g., pp. 31, 120, 349]. Zeldes [38] found, by means of computer simulations, that the presence of idiosyncratic risk makes consumption concave. There is also strong empirical evidence for concavity (see, e.g., $[22,36]$ ). Many papers have been devoted to the investigation of a diminishing marginal propensity to consume in standard, macroeconomic models without risky assets, see, e.g., [1,2,17,18]. Notably, Carroll and Kimball [2] prove that consumption is a concave function of wealth for HARA utilities when only one period risk free bonds are available for trading. Proposition 5.17 establishes a much stronger result. Namely, it shows that the concavity holds for a the whole class $\mathfrak{C}$ of incomplete markets and for a large class of utitlities, including HARA as a small subclass, and that consumption is in fact jointly concave in wealth and endowment. Note that concavity in the whole endowment process is a much stronger fact than concavity just in wealth.

Levhari and Srinivasan [20] and Rotschild and Stiglitz [31] analyzed how optimal savings and portfolio composition depend on the riskiness of the underlying assets. In particular, they proved a surprising result that, for a CRRA utiliy, optimal savings increase (decrease) in the size of uncertainty when risk aversion is larger (smaller) than one. These phenomena can also be interpreted as precautionary savings effects, but they are generated by the uncertainty in asset returns, and not by the income uncertainty. We do not analyze such effects in this paper. See, Kimball [18] for a general theory of precautionary portfolio composition in one period models.

Gollier [12, Proposition 74] compares precautionary savings in a one period model with only a risk free bond with savings in a complete market model and obtains conditions, under which market completeness increases savings. In an attempt to generalize this result to incomplete markets with risky assets, Gollier [12, Proposition 75] considers an artificial model with a risky asset that only lives in the second period. That is, optimal investment in this risky asset is not intertemporal. He shows that the above result for complete markets extends to this artificial model. Unfortunately, his method of proof does not work for incomplete market models with intertemporal risky assets. It is possible to show, using our methods, that Gollier's result extends to the class $\mathfrak{C}$ of incomplete markets and to any number of periods. This will be done in a separate paper.

In this paper we work exclusively in discrete time. Using our quantitative control over consumption streams, it is possible to pass to the continuous time limit. All the beautiful recursive structure disappears, but the important properties (such as convexity) remain. This will 
be done in a separate paper. There is a large literature, initiated by Merton [26], devoted to continuous time models. The main idea is to reformulate the problem in terms of a Hamilton-Jacobi-Bellman, nonlinear partial differential dynamic programming equation, and then solve it explicitly for very special choices of utility functions, endowment processes and asset prices. For the most part, incompleteness is modelled phenomenologically through an exogenous diffusion process, see, e.g, $[4,6,9,14,26]$. There are other special methods for logarithmic utility functions, see [11].

A brief outline of the paper

In Sect. 2, we introduce two new notions for general incomplete markets, the payoff subspaces and the aggregate state price densities. We show how budget constraints and first order conditions can be written down in an elegant and clear way using these new notions. Using the structure of the budget constraints and first order conditions, we show in Theorem 2.15 that both marginal propensity to consume and marginal propensity to save are positive for any incomplete market and any utility function.

In Sect. 3 we introduce the class $\mathfrak{C}$ of incomplete markets and give its characterization in terms of natural positivity properties.

In Sect. 4 we present three very important classes of incomplete markets, idiosyncratically incomplete, discrete time diffusion driven and markets with only one period risk free bonds, and show that they are subclasses of $\mathfrak{C}$.

In Sect. 5 we study the recursive structure of the optimal consumption streams for markets in $\mathfrak{C}$ and use this structure to derive important economic properties of precautionary savings and marginal propensity to consume.

Finally, in Sect. 6 we derive an approximation to the optimal consumption stream with CRRA utility when idiosyncratic risk is small. Our approximate optimal consumption streams are crucial for our analysis of equilibria in [23].

\section{General incomplete markets}

\subsection{The structure of market incompleteness}

The uncertainty in our model is captured by a finite, filtered probability space $(\Omega, \mathscr{G}, \mathscr{B})$ where the filtration $\mathscr{G}=\left(\mathscr{G}_{t}\right)_{t=0}^{T}$ satisfies

$$
\{\emptyset, \Omega\}=\mathscr{G}_{0} \subset \mathscr{G}_{1} \subset \cdots \subset \mathscr{G}_{T}=\mathscr{B}
$$

There are $T$ time periods. We emphasize that everywhere in this paper the probability space $\Omega$ and time horizon $T$ are assumed to be finite. All the remarkable recursive structure of the optimal consumption streams that we uncover disappears for infinite state spaces. But, because we have a quantitative control over the optimal streams, we can pass to infinite state space, infinite horizon and continuous time limits. The structure disappears, but most important economic properties (such as convexity and uniform bounds) are preserved in the limit.

We allow for an arbitrary type of market incompleteness, except for a natural

Assumption 1 One period risk free bonds are available for trading at each moment of time.

Definition 2.1 Let $\mathscr{M}=\left\{A_{1}, \ldots, A_{N}\right\}$ be the underlying incomplete market with financial assets $A_{1}, \ldots, A_{N}$. An asset $A_{i}$ has a price process $\left(p_{i t}\right)$ and a dividend process $\left(d_{i t}\right)$. The 
payoff subspace $\mathscr{L}_{t}$ at time $t$ is defined by

$$
\mathscr{L}_{t}=\left\{\sum_{i=1}^{N} x_{i, t-1}\left(p_{i t}+d_{i t}\right) \mid x_{i, t-1} \in L_{2}\left(\mathscr{G}_{t-1}\right) \forall i\right\}
$$

This is the set of payoffs at time $t$ of all possible (of course, $\mathscr{G}_{t-1}$-measurable) investments at time $t-1$. We denote by $\mathrm{P}_{\mathscr{L}}^{t}$ the orthogonal projection onto the subspace $\mathscr{L}_{t}$ in the space $L_{2}\left(\mathscr{G}_{t}\right)$. Similarly, let $\mathrm{P}_{\mathscr{G}}^{t}, t=1, \ldots, T$, be the orthogonal projection (conditional expectation) from $L_{2}(\Omega, \mathscr{B})$ onto $L_{2}\left(\Omega, \mathscr{G}_{t}\right)$. We write $\mathbf{P}_{\mathscr{G}}$ for the direct sum

$$
\mathbf{P}_{\mathscr{G}}=\bigoplus_{t=1}^{T} \mathrm{P}_{\mathscr{G}}^{t}
$$

Note that, by Assumption $1, L_{2}\left(\mathscr{G}_{t-1}\right) \subset \mathscr{L}_{t}$ and $\mathrm{P}_{\mathscr{L}}^{t} 1=1$. Furthermore, for any $\mathscr{G}_{t-1^{-}}$ measurable $Y$ and any $\mathscr{G}_{t}$-measurable $X$ we have

$$
\mathrm{P}_{\mathscr{L}}^{t} X Y=Y \mathrm{P}_{\mathscr{L}}^{t} X
$$

The projection $\mathrm{P}_{\mathscr{L}}^{t}$ is very important for understanding the structure of market incompleteness.

A portfolio strategy for an agent, with a $\mathscr{G}$ adapted individual endowment process, trading on the market $\mathscr{M}$ is an $n$ dimensional, $\mathscr{G}$ adapted process $\mathbf{x}=\left(\mathbf{x}_{1}, \ldots, \mathbf{x}_{n}\right)$ Here, $\mathbf{x}_{j}=$ $\left(x_{j 0}, \ldots, x_{j T-1}, 0\right)$. The random variable $x_{j t}$ counts the number of shares of asset $A_{j}$ held at time $t+1$ before dividends are paid and assets are traded. The last component 0 formalizes the convention that no investments are made at the final time period $T$.

Definition 2.2 The dividend process $\mathbf{D}_{\mathbf{x}}$ generated by the portfolio strategy $\mathbf{x}$ is

$$
D_{\mathbf{x}, t}=\sum_{j=1}^{N}\left(d_{j t}+p_{j t}\right) x_{j t-1}-\sum_{j=1}^{N} p_{j t} x_{j t}
$$

for $t=0, \ldots, T$, where $\mathbf{d}_{j}$ and $\mathbf{p}_{j}$ are the dividend and price processes of the asset $A_{j}$. In particular, the initial investment is $D_{\mathbf{x}, 0}=-\sum_{j=1}^{N} p_{j 0} x_{j 0}$,

Recall the standard definition of state price densities.

Definition 2.3 A $\mathscr{G}$-adapted process $\mathbf{R}=\left(R_{t}\right)$ is referred to as a state price density process (SPD process) for the market $\mathscr{M}$ if the identity

$$
R_{t} p_{i t}=E\left[R_{t+1}\left(p_{i t+1}+d_{i t+1}\right) \mid \mathscr{G}_{t}\right]
$$

holds for any asset $A_{i}, i=1, \ldots, N$, and any $t=0, \ldots, T-1$. In particular, under the standard no-bubble condition $p_{i T}=0$, the price

$$
p_{i t}=R_{t}^{-1} E\left[\sum_{\tau=1}^{T-t} R_{t+\tau} d_{i t+\tau} \mid \mathscr{G}_{t}\right]
$$

is the discounted value of future dividends.

State price densities are dual objects to asset prices. They allow formulating many issues in a very elegant and clear way. We recall some well known properties of state price densities. 
Lemma 2.4 There is no arbitrage in the market if and only if there exists a positive state price density process.

The market is dynamically complete if and only if there exists a unique, positive state price density process.

A process $\mathbf{D}$ is a dividend process of a portfolio strategy if and only if it is orthogonal to any SPD process, i.e.,

$$
E\left[\sum_{t=0}^{T} D_{t} R_{t}\right]=0
$$

for any SPD process $\mathbf{R}[7]$.

When markets are incomplete, there are infinitely many state price density processes. This is one of the main difficulties in the analysis of utility maximization in incomplete markets. It turns out that there is a unique, natural, "aggregate" state price density process such that all budget constraints and first order conditions can be formulated in terms of this special process.

Lemma 2.5 Under the assumption of no arbitrage, there exists a unique, aggregate state price density process $\mathbf{M}=\left(M_{t}\right)$ such that $M_{t} \in \mathscr{L}_{t}$ for all $t=1, \ldots, T$. Furthermore, $a$ process $\mathbf{R}=\left(R_{t}\right)$ is a state price density process if and only if

$$
\mathrm{P}_{\mathscr{L}}^{t} \frac{R_{t}}{R_{t-1}}=\frac{M_{t}}{M_{t-1}}
$$

for all $t$.

The aggregate SPD process $\mathbf{M}$ is natural because it lives in the market subspace, just like the prices themselves. Note that, in general, $\mathbf{M}$ is not positive. This is the main source of problems: the projection $\mathrm{P}_{\mathscr{L}}^{t}$ is not necessarily positivity preserving. We will come back to this point later (see, Sect. 3).

\subsection{The optimal consumption stream}

In this section we will use the aggregate SPD process $\mathbf{M}$ to write down the first order conditions and budget constraints solely in terms of $\mathbf{M}$.

Definition 2.6 The budget set $\mathrm{B}(\mathbf{w})$ of an agent with a $\mathscr{G}$-adapted endowment process $\mathbf{w}=$ $\left(w_{t}\right)$ is given by

$$
\mathrm{B}(\mathbf{w}):=\left\{\mathbf{C}=\mathbf{w}+D_{\mathbf{x}}>0 \mid \mathbf{x} \text { is a } \mathscr{G} \text { adapted portfolio strategy }\right\}
$$

Lemma 2.7 Let $\mathbf{M}=\left(M_{t}\right)$ be the aggregate state price density process for the market $\mathscr{M}$. A positive consumption stream $\mathbf{C}=\left(c_{t}, t=0, \ldots, T\right)$ belongs to $\mathrm{B}(\mathbf{w})$ if and only if

$$
\left(I-\mathrm{P}_{\mathscr{L}}^{t}\right) \mathrm{P}_{\mathscr{G}}^{t}\left[\sum_{\tau=t}^{T}\left(c_{\tau}-w_{\tau}\right) \frac{M_{\tau}}{M_{t}}\right]=0
$$

for all $t=1, \ldots, T$ and

$$
E\left[\sum_{\tau=0}^{T}\left(c_{\tau}-w_{\tau}\right) M_{\tau}\right]=0
$$


Note that (2.4) is the standard, complete market budget constraint. Constraints (2.3) are new and reflect market incompleteness. They simply mean that the financial wealth

$$
\mathrm{P}_{\mathscr{G}}^{t}\left[\sum_{\tau=t}^{T}\left(c_{\tau}-w_{\tau}\right) \frac{M_{\tau}}{M_{t}}\right]=\sum_{j=1}^{N}\left(d_{j t}+q_{j t}\right) x_{j t-1}
$$

at time $t$ must belong to the payoff subspace $\mathscr{L}_{t}$ for each $t$. We will need the following important quantities.

Definition 2.8 Given a $\mathscr{G}$ adapted endowment process $\mathbf{w}$, let for each $t=1, \ldots, T$

$$
\mathrm{Y}_{t}(\mathbf{w}, \mathbf{M})=M_{t}^{-1} E\left[\sum_{\tau=t}^{T} w_{\tau} M_{\tau} \mid \mathscr{G}_{t}\right]
$$

be the value at time $t$ of the future stream of endowment and

$$
\mathrm{I}_{t}(\mathbf{w}, \mathbf{M})=\left(I-\mathrm{P}_{\mathscr{L}}^{t}\right) \mathrm{Y}_{t}
$$

be the unhedgeable component of the value of the endowment stream.

Consider an agent with a $\mathscr{G}$-adapted endowment process $\mathbf{w}$, trading in the market to maximize

$$
E\left[\sum_{t=0}^{T} e^{-\rho t} u\left(c_{t}\right)\right]
$$

over all consumption streams $\mathbf{C}=\left(c_{t}\right)$ in the budget set $B(\mathbf{w})$. Here, $u(c)$ is a monotone increasing, concave utility function satisfying the Inada conditions

$$
\lim _{c \rightarrow 0} u^{\prime}(c)=+\infty, \quad \lim _{c \rightarrow+\infty} u^{\prime}(c)=0
$$

Since, by assumption, $\Omega$ is finite, Inada conditions and strict concavity guarantee existence and uniqueness of an optimal consumption stream $\mathbf{C}=\left(c_{t}\right)$ satisfying the standard Euler equation

$$
u^{\prime}\left(c_{t}\right) p_{i t}=E\left[e^{-\rho} u^{\prime}\left(c_{t+1}\right)\left(p_{i t+1}+d_{i t+1}\right) \mid \mathscr{G}_{t}\right]
$$

for any asset $A_{i}, i=1, \ldots, N$. Thus, Lemma 2.5 implies

Proposition 2.9 The optimal consumption stream $\mathbf{C}=\left(c_{t}\right)$ is uniquely determined by the first order conditions

$$
\mathrm{P}_{\mathscr{L}}^{t+1} e^{-\rho} u^{\prime}\left(c_{t+1}\right)=\frac{M_{t+1}}{M_{t}} u^{\prime}\left(c_{t}\right)
$$

and the budget constraints (2.3) and (2.4).

Equations (2.5), (2.3) and (2.4) form a highly non linear and complicated system of equations. We would like to have a recursive procedure, allowing us to construct the solution and derive its properties. We will need several definitions. 
Definition 2.10 Fix a time period $t$. A map $F: \mathscr{L}_{t} \rightarrow \mathscr{L}_{t}$ is monotone decreasing conditioned on $\mathscr{G}_{t-1}$ if

$$
E\left[(F(X)-F(Y))(X-Y) \mid \mathscr{G}_{t-1}\right]<0
$$

for all $X \neq Y \in \mathscr{L}_{t}$.

Note that, obviously, a monotone decreasing map is injective and its inverse is also monotone decreasing.

We will now construct the optimal consumption stream recursively. The construction involves the notion of a random function. The random functions arising below may have complicated domains of definitions, but we do not discuss it here (see, Sect. 5.2). Given a sigma algebra $\mathfrak{A}$, an $\mathfrak{A}$-measurable random function is just an $\mathfrak{A}$-measurable map from the probability space to the set of smooth functions. ${ }^{1}$ Similarly, we will also consider random maps, mapping real numbers into random variables in $\mathscr{L}_{t}$.

We will need the following auxiliary

Lemma 2.11 Let $f(s, x): \Omega \times \mathbb{R} \rightarrow \mathbb{R}$ be a $\mathscr{G}_{t}$ measurable random function. Then, the map $J: \mathscr{L}_{t} \rightarrow \mathscr{L}_{t}$ defined via

$$
J(X)=\mathrm{P}_{\mathscr{L}}^{t}\left(u^{\prime}(f(s, X))\right)
$$

is monotone decreasing conditioned on $\mathscr{G}_{t-1}$ (see, Definition 2.10). Furthermore, for any $Y \in \mathscr{L}_{t}$, the $\mathscr{G}_{t-1}$-measurable random function

$$
K(s, x)=\mathrm{P}_{\mathscr{G}}^{t-1}\left[Y J^{-1}(Y x)\right]
$$

is monotone decreasing in $x$.

Note that $F(s, x)=J^{-1}(Y x)$ is a random map $\mathbb{R} \rightarrow \mathscr{L}_{t}$, but $K(s, x)$ is a random function.

Of course, one must be careful here with the domains of definition. But, since we know a-priori that the optimal consumption stream exists, everything is already in the domain of definition and we do not have to worry about it. One easily checks that the derivatives of all monotone maps in question are invertible (in fact, they are positive definite) and therefore, the inverse function theorem guarantees that the inverse map is well defined in an open neighborhood. Of course, these questions are important if we want to construct a numerical agorithm. See Sect. 5.2 for a detailed discussion of these questions for the class $\mathfrak{C}$ of incomplete markets.

We will also need

Definition 2.12 Let for each $t=1, \ldots, T$

$$
V_{t}(\mathbf{w}, \mathbf{M}):=\mathrm{P}_{\mathscr{L}}^{t}\left[M_{t}^{-1} \sum_{\tau=t}^{T} c_{\tau}(\mathbf{w}, \mathbf{M}) M_{\tau}\right]
$$

This quantity

$$
V_{t}(\mathbf{w}, \mathbf{M}):=\sum_{j=1}^{N}\left(d_{j t}+p_{j t}\right) x_{j t-1}+\mathrm{P}_{\mathscr{L}}^{t}\left[M_{t}^{-1} \sum_{\tau=t}^{T} w_{\tau} M_{\tau}\right]
$$

is the financial wealth plus the market value of the future endowment stream.

${ }_{1}^{1}$ Since our probability space $\Omega$ is finite, we do not have to discuss technical issues of measurability of a map into an infinite dimensional space. 
For each endowment process $\mathbf{w}$, set

$$
G_{T}(s, \mathbf{w})(x):=\left(\mathrm{I}_{T}(\mathbf{w}, \mathbf{M})+x\right)
$$

By construction, it is a $\mathscr{G}_{T}$ measurable random function. Lemma 2.11 implies that there exists a $\mathscr{G}_{T}$-measurable random map $F_{T}(s, \mathbf{w})(x): \mathbb{R} \rightarrow \mathscr{L}_{T}$, being the unique solution to the equation

$$
e^{-\rho} \mathrm{P}_{\mathscr{L}}^{T}\left[u^{\prime}\left(G_{T}(s, \mathbf{w})\left(F_{T}(x)\right)\right)\right]=u^{\prime}(x) M_{T} M_{T-1}^{-1}
$$

Continuing inductively, we obtain

Proposition 2.13 Fix a nonnegative endowment process $\mathbf{w}$. Let $G_{T}$ and $F_{T}$ be the random functions (maps) given by (2.6) and (2.7). For each $t=1, \ldots, T-1$ there exists a pair of $\mathscr{G}_{t}$-measurable random functions $G_{t}(s, \mathbf{w})(x)$ (taking values in $\mathbb{R}$ ) and $F_{t}(s, \mathbf{w})(x)$ (taking values in $\mathscr{L}_{t}$ ). They are inductively determined as the unique solutions (recall, Definition 2.8) to the equations

$$
G_{t}(x)+E\left[\frac{M_{t+1}}{M_{t}} F_{t+1}(s, \mathbf{w})\left(G_{t}(x)\right) \mid \mathscr{G}_{t}\right]=x+\mathrm{I}_{t}
$$

and

$$
e^{-\rho} \mathrm{P}_{\mathscr{L}}^{t}\left[u^{\prime}\left(G_{t}(s, \mathbf{w})\left(F_{t}(x)\right)\right)\right]=u^{\prime}(x) M_{t} M_{t-1}^{-1}
$$

Finally, $G_{0}$ is the unique solution to

$$
G_{0}(x)+E\left[M_{1} F_{1}(s, \mathbf{w})\left(G_{0}(x)\right) \mid \mathscr{G}_{t}\right]=x+E\left[\sum_{t=1}^{T} w_{t} M_{t}\right]
$$

For every state $s \in \Omega$ and every nonnegative $\mathbf{w}$ the random functions $G_{t}, t=0, \ldots, T$, and

$$
K_{t}(s, x)=E\left[\frac{M_{t+1}}{M_{t}} F_{t+1}(s, \mathbf{w})(x) \mid \mathscr{G}_{t}\right]
$$

$($ for $t \geqslant 1)$ are monotone increasing in $x$.

The optimal consumption stream is constructed recursively in

Theorem 2.14 Let $\mathbf{C}(\mathbf{w}, \mathbf{M})=\left(c_{t}, t=1, \ldots, T\right)$ be the optimal consumption stream. For every state $s \in \Omega$, and all $t=1, \ldots, T$,

$$
V_{t}=F_{t}(s, \mathbf{w})\left(c_{t-1}\right)
$$

and

$$
c_{t}=G_{t}(s, \mathbf{w})\left(V_{t}\right)
$$

Finally, $c_{0}=G_{0}(s, \mathbf{w})\left(w_{0}\right)$. Here, $F_{t}, G_{t}, t=1, \ldots, T$, are the random functions constructed in Proposition 2.13.

The monotonicity results of Proposition 2.13 imply

Theorem 2.15 The marginal propensity to consume

$$
\frac{\partial c_{t}}{\partial V_{t}}
$$


and the marginal propensity to save

$$
1-\frac{\partial c_{t}}{\partial V_{t}}
$$

are positive for each $t=1, \ldots, T$. Similarly, both marginal propensities

$$
\frac{\partial c_{0}}{w_{0}} \text { and } 1-\frac{\partial c_{0}}{\partial w_{0}}
$$

are also positive.

\section{The class $\mathfrak{C}$ of incomplete markets}

The absence of arbitrage is equivalent to the existence of a positive SPD process $\mathbf{R}$. But, even though there exists a positive $\mathbf{R}$, it may happen that

$$
\mathrm{P}_{\mathscr{L}}^{t} R_{t}=R_{t-1} \frac{M_{t}}{M_{t-1}}
$$

takes negative values and, consequently, the aggregate SPD process $\mathbf{M}$ may take negative values. Thus, it is crucial for us to know when the projection $\mathrm{P}_{\mathscr{L}}^{t}$ is positivity preserving. There is another natural reason why an economically reasonable $\mathrm{P}_{\mathscr{L}}^{t}$ should be positivity preserving. This is the so-called minimal variance hedging. The following simple fact is true:

Proposition 3.1 Let $X$ be a random claim at time t. Then,

$$
\mathrm{P}_{\mathscr{L}}^{t} X=\arg \min \left\{E\left[|X-Y|^{2}\right] \mid Y \in \mathscr{L}_{t}\right\}
$$

That is, the projection $\mathrm{P}_{\mathscr{L}}^{t} X$ is the payoff of the portfolio, that best approximates the claim $Y$ in the $L_{2}$-distance.

Naturally, one would like to generate a positive payoff by hedging a positive outcome. Amazingly, the class of incomplete markets for which $\mathrm{P}_{\mathscr{L}}^{t}$ is positivity preserving turns out to have numerous remarkable economic properties. Furthermore, to our own surprise, it includes almost all examples of market incompleteness known in finance and macroeconomics.

Theorem 3.2 The projection $\mathrm{P}_{\mathscr{L}}^{t}$ is positivity preserving (that is, it maps nonnegative random variables to nonnegative ones) if and only if there exists a sigma algebra $\mathfrak{A}$ with $\mathscr{G}_{t-1} \subset \mathfrak{A} \subset$ $\mathscr{G}_{t}$ such that

$$
\mathrm{P}_{\mathscr{L}}^{t} X=E[X \mid \mathfrak{A}]
$$

and $\mathscr{L}_{t}=L_{2}(\mathfrak{A})$. That is, $\mathrm{P}_{\mathscr{L}}^{t}$ is positivity preserving if and only if it is a conditional expectation.

Now we are ready to introduce the class $\mathfrak{C}$ of incomplete markets.

Definition 3.3 An incomplete market $\mathscr{M}$ belongs to the class $\mathfrak{C}$ if there exists a subfiltration $\mathscr{H}=\left(\mathscr{H}_{t}\right)$ of $\mathscr{G}$ such that

- $\mathscr{H}_{t+1} \supset \mathscr{G}_{t} \supset \mathscr{H}_{t}$ for all $t$.

- The payoff process $\left(p_{i t}+d_{i t}\right)$ of any asset $A_{i}$ is adapted to $\mathscr{H}$. 
- Any $\mathscr{H}_{t}$ measurable claim $Y$ can be replicated by a $\mathscr{G}_{t-1}$ measurable portfolio $\pi_{1}, \ldots, \pi_{N}$ of assets, purchased at time $t-1$. That is,

$$
Y=\sum_{i=1}^{T} \pi_{i}\left(p_{i t}+d_{i t}\right)
$$

We refer to $\mathscr{H}$ as the hedgeable filtration.

Theorem 3.2 provides a remarkable characterization of the class $\mathfrak{C}$ :

Proposition 3.4 An incomplete market belongs to the class $\mathfrak{C}$ if and only if $\mathrm{P}_{\mathscr{L}}^{t}$ is positivity preserving for any $t=1, \ldots, T$.

The aggregate state price density process $\mathbf{M}$ is positive and is the unique state price density process adapted to the hedgeable filtration $\mathscr{H}$.

We also mention an interesting connection with the notion of the minimal martingale measure, introduced by Schweizer [33]. It is not difficult to show, using the minimal entropy property (see [34]), that, for incomplete markets in the class $\mathfrak{C}$, the aggregate SPD process corresponds to the minimal martingale measure.

\section{Examples of incomplete markets in the class $\mathfrak{C}$}

\subsection{Idiosyncratic incompleteness}

In this section we describe a special type of incomplete markets, for which the only source of incompleteness is idiosyncratic income risk. Pure idiosyncratic incompleteness is characterized by the following properties.

\section{Definition 4.1 Idiosyncratic incompleteness.}

- There exist two filtrations $\mathscr{F}=\left(\mathscr{F}_{t}, t=0, \ldots, T\right)$ and $\mathscr{G}=\left(\mathscr{G}_{t}, t=0, \ldots, T\right)$ of the underlying sigma algebra $\mathscr{B}$ satisfying $\mathscr{G}_{t} \supset \mathscr{F}_{t}$ for each $t=0, \ldots, T$.

- Aggregate filtration $\mathscr{F}$ contains information only about "aggregate" events.

- The dominating, idiosyncratic filtration $\mathscr{G}$ contains additional information about individuals, that is, idiosyncratic events.

- All asset prices and dividends are adapted to $\mathscr{F}$. That is, asset prices do not care about idiosyncratic events.

- The market is dynamically complete with respect to the aggregate filtration $\mathscr{F}$ (but not with respect to $\mathscr{G}$ ). That is, idiosyncratic risk is the only source of incompleteness.

The following example illustrates the structure of idiosyncratic incompleteness.

Example There is only one risky asset (equity) with binomial dividend process and one period risk free bonds are traded at every period. Then, the aggregate filtration $\mathscr{F}$ is simply the binomial filtration and it is well known that the market is dynamically complete. But, there is a larger, idiosyncratic filtration $\mathscr{G}$ and idiosyncratic income processes of agents are adapted to $\mathscr{G}$ (but not to $\mathscr{F}$ ). A recursive structure, similar to ours, has been discovered in the binomial case by Musiela and Zariphopoulou [30], Musiela and Zariphopoulou [29].

Utility maximization problems in a continuous time version of this market have been considered by Duffie et al. [6] and Hendersen [14]. 
The main result of this section is

Proposition 4.2 Any idiosyncratically incomplete market belongs to the class $\mathfrak{C}$. The hedgeable sigma algebra $\mathscr{H}_{t}$ is given by

$$
\mathscr{H}_{t}=\sigma\left(\mathscr{F}_{t}, \mathscr{G}_{t-1}\right)
$$

That is, $\mathscr{H}_{t}$ is the minimal sigma algebra, containing $\mathscr{F}_{t}$ and $\mathscr{G}_{t-1}$.

It is clear that the difference between $\mathscr{H}_{t}$ and $\mathscr{G}_{t}$ constitutes exactly the unhedgeable idiosyncratic events. Because the market is complete with respect to $\mathscr{F}$, we can always hedge against the events in $\mathscr{H}_{t}$ by a $\mathscr{G}_{t-1}$ measurable investment at time $t-1$. But, since all asset returns are $\mathscr{F}$ adapted, we can not hedge against anything in $\mathscr{G}_{t} \backslash \mathscr{H}_{t}$. Therefore, given a $\mathscr{G}_{t}$-measurable shock $Y_{t}$ at time $t$, the projection

$$
Y_{t}-\mathrm{P}_{\mathscr{H}}^{t} Y_{t}=Y_{t}-E\left[Y_{t} \mid \mathscr{H}_{t}\right]
$$

is precisely the unhedgeable part of $Y_{t}$.

The class of idiosyncratically incomplete markets plays a very important role in macroeconomics, see, e.g., Constantinides and Duffie [3]. We are very lucky that all idiosyncratically incomplete markets fit into the class $\mathfrak{C}$ !

It will be technically useful to assume that knowledge of idiosyncratic events at time $t$ does not give us any additional information about aggregate events at time $t+1$. We make the formal

Assumption 2 For each $t=1, \ldots, T$, and for every integrable random variable $Y$ measurable with respect to $\mathscr{F}_{t+1}$,

$$
E\left[Y \mid \mathscr{F}_{t}\right]=E\left[Y \mid \mathscr{G}_{t}\right]
$$

Note that, since the market is complete with respect to $\mathscr{F}$, there exists a unique, SPD process $\mathbf{M}$ adapted to $\mathscr{F}$ such that

$$
M_{t} p_{i t}=E\left[M_{t+1}\left(p_{i t+1}+d_{i t+1}\right) \mid \mathscr{F}_{t}\right]
$$

holds for any asset $A_{i}, i=1, \ldots, N$. But, the identity

$$
M_{t} p_{i t}=E\left[M_{t+1}\left(p_{i t+1}+d_{i t+1}\right) \mid \mathscr{G}_{t}\right]
$$

does not hold in general. Assumption 2 fixes this problem.

Proposition 4.3 Under Assumption 2, the unique, aggregate SPD process $\mathbf{M}$ adapted to $\mathscr{H}$ is, in fact, adapted to $\mathscr{F}$ and coincides with the unique SPD process relative to $\mathscr{F}$.

\subsection{Discrete time diffusion driven incompleteness}

Diffusion driven incomplete market models have become standard in modern finance. In this section we introduce a class of discrete time diffusion driven incomplete markets and show that they all belong to the class $\mathfrak{C}$.

Let $e_{0}=(1,1, \ldots, 1)$ be the "riskless vector" and $\left\{e_{0}, e_{1}, \ldots, e_{N}\right\} \subset \mathbb{R}^{N+1}$ be an orthonormal basis of $\mathbb{R}^{N+1}$. That is,

$$
\left\langle e_{i}, e_{j}\right\rangle=\sum_{k=1}^{N+1} e_{i k} e_{j k}=\delta_{i j}
$$


where $\delta_{i j}$ is the Dirac delta. Let $\omega=\left\{s_{1}, \ldots, s_{N+1}\right\}$ be an $N+1$-point probability space with each state having probability $1 /(N+1)$. Let $X_{i}, i=1, \ldots, N$, be the random variable taking value $e_{i j}$ in the state $s_{j}$.

Consider a sequence $\left(X_{1 t}, \ldots, X_{N t}\right), t \geqslant 1$, of independent, identically distributed copies of the random vector $\left(X_{1}, \ldots, X_{N}\right)$. Obviously,

$$
E\left[X_{i t} X_{j t}\right]=\delta_{i j} \quad \text { and } \quad E\left[X_{i}\right]=0
$$

for all $i, j=1, \ldots, N$.

Definition 4.4 The $N$-dimensional process $\mathbf{B}_{t}=\left(B_{1 t}, \ldots, B_{N t}\right)$ defined by $\mathbf{B}_{0}=0$ and

$$
B_{i t+1}=B_{i t}+X_{i t}
$$

is referred to as a discrete time $N$-dimensional Brownian motion.

It is well known (see, e.g., [13]) that an $N$-dimensional Brownian motion can in fact be approximated by such discrete time processes. The most important property of this discrete time Brownian motion is that the spanning property is preserved: the vectors $e_{i}, i=0, \ldots, N+1$ form a basis for $\mathbb{R}^{N+1}$. Now we are ready to make the

Definition 4.5 Let $\mathscr{G}$ be the underlying filtration. A discrete time, diffusion driven incomplete market model is given by $N$ risky asset price processes $\left(P_{i}(t), i=1, \ldots, N\right)$ defined by

$$
\log \left(P_{i}(t+1)\right)-\log \left(P_{i}(t)\right)=\mu_{i}(s, t)+\sum_{j=1}^{N} \sigma_{i j}(s, t)\left(B_{j t+1}-B_{j t}\right)
$$

where $\left(B_{1 t}, \ldots, B_{N t}\right)$ is a discrete time $N$-dimensional Brownian motion and $\mu_{i}$, $i=1, \ldots, N$, and $\sigma_{i j}, i=1, \ldots, N$, are arbitrary, $\mathscr{G}$-adapted processes, such that the matrix is nondegenerate for a.e. $s$ and every $t$.

The spanning property of the discrete time Brownian motion immediately implies the following

Proposition 4.6 Any discrete time, diffusion driven incomplete market model belongs to the class $\mathfrak{C}$. The corresponding hedgeable filtration $\mathscr{H}$ is given by

$$
\mathscr{H}_{t}=\sigma\left(\mathscr{F}_{t}, \mathscr{G}_{t-1}\right)
$$

where $\mathscr{F}=\left(\mathscr{F}_{t}\right)$ is the aggregate filtration, generated by the discrete time Brownian motion $\left(B_{1}, \ldots, B_{N}\right)$.

Note that $\mu_{i}$ and $\sigma_{i j}$ are allowed to be arbitrary $\mathscr{G}$-adapted processes. A natural continuous time analog of the stochastic difference equations (4.2) are the equations

$$
\frac{d P_{i}}{P_{i}}=\mu_{i}(s, t) d t+\sum_{j=1}^{N} \sigma_{i j}(s, t) d B_{j t}
$$

where $\left(B_{1}, \ldots, B_{N}\right)$ is an $N$-dimensional Brownian motion. The class of models, described by (4.3), looks more restrictive than the class of all standard, diffusion driven incomplete market models

$$
\frac{d P_{i}}{P_{i}}=\mu_{i}(s, t) d t+\sum_{j=1}^{D} \sigma_{i j}(s, t) d B_{j t}
$$


where $D>N$ is the dimension of the driving Brownian motion (all popular stochastic volatility models, such as, e.g., the model of Heston [15] fit into this class). The following proposition shows that, in fact, the two classes coincide.

Proposition 4.7 Suppose that the rank of the $N \times D$ matrix $\sigma=\left(\sigma_{i j}\right)_{i, j=1}^{N, D}$ in (4.4) equals $N$ almost surely for all $t$. Then, there exists an $N$-dimensional Brownian motion $\left(W_{1}, \ldots, W_{N}\right)$ and a $\mathscr{G}$-adapted matrix-valued process $\beta=\left(\beta_{i j}\right)_{i j=1}^{N}$ such that the system of SDE's (4.4) is equivalent to the system

$$
\frac{d P_{i}}{P_{i}}=\mu_{i}(s, t) d t+\sum_{j=1}^{N} \beta_{i j}(s, t) d W_{j t}
$$

Thus, any model of the form (4.4) can be rewritten in the form (4.3).

Proof Let $\sigma^{\prime}$ be the transpose of $\sigma$. The process

$$
\mathbf{W}_{t}=\int_{0}^{t}\left(\sigma \sigma^{\prime}\right)^{-1 / 2} \sigma d \mathbf{B}
$$

is a continuous, square integrable martingale with the quadratic covariance matrix $t I d_{N}$. The classical theorem of $\mathrm{P}$. Levy says that $\mathbf{W}$ is an $N$-dimensional Brownian motion. The matrix

$$
\beta=\left(\sigma \sigma^{\prime}\right)^{1 / 2}
$$

is the required.

The natural impulse would be to take an arbitrary continuous time diffusion driven model, discretize it, get all the nice properties of the optimal consumption stream, and then pass to the continuous time limit. But, proving convergence is not straightforward. He [13] shows that for Markov processes the prices weakly converge to their continuous time limit. In general, proving convergence is quite technical and we will do this in a subsequent paper, see, e.g., Willinger and Taqqu [37], Duffie and Protter [8] for some work in this direction. After all, for practical applications, both in real world and in computer simulations, time is discrete.

\subsection{The market with only one period risk free bonds}

In the standard, macroeconomic saving problem, one period risk free bonds are the only assets available for trading. This incomplete market obviously belongs to the class $\mathfrak{C}$ with the hedgeable filtration

$$
\mathscr{H}_{t}=\mathscr{G}_{t-1}
$$

for all $t$. Furthermore, the aggregate state price density process is in this case given by

$$
M_{t}=r_{1}^{-1} \cdots r_{t}^{-1}
$$

where $r_{t}$ is the risk free rate at time $t$.

\section{The optimal consumption stream for an incomplete market in the class $\mathfrak{C}$}

The nice structure of markets in the class $\mathfrak{C}$ allows us to write the budget constraints and first order conditions in a very elegant way. Lemma 2.7 takes the form 
Proposition 5.1 Let $\mathscr{M} \in \mathfrak{C}$, $\mathscr{H}$ the corresponding hedgeable filtration and $\mathbf{M}$ the aggregate state price density process. A consumption stream $\mathbf{C}=\left(c_{t}, t=0, \ldots, T\right)$ lies in the budget set $\mathrm{B}(\mathbf{w})$ if and only if

$$
E\left[\sum_{\tau=0}^{T}\left(c_{\tau}-w_{\tau}\right) M_{\tau}\right]=0
$$

and

$$
E\left[\sum_{\tau=t}^{T}\left(c_{\tau}-w_{\tau}\right) M_{\tau} \mid \mathscr{H}_{t}\right]=E\left[\sum_{\tau=t}^{T}\left(c_{\tau}-w_{\tau}\right) M_{\tau} \mid \mathscr{G}_{t}\right]
$$

for all $t=1, \ldots, T$. Here, $\mathbf{M}=\left(M_{t}\right)$ is the aggregate state price density process.

Similarly, Proposition 2.9 takes the form

Proposition 5.2 Let $\mathscr{H}$ be the hedgeable filtration and $\mathbf{M}$ the aggregate state price density process. The optimal consumption stream is uniquely determined by the budget constraints of Lemma 5.1 and the Euler equations

$$
E\left[e^{-\rho} u^{\prime}\left(c_{t+1}\right) M_{t+1}^{-1} \mid \mathscr{H}_{t+1}\right]=u^{\prime}\left(c_{t}\right) M_{t}^{-1}
$$

That is, the quotient $e^{-\rho t} u^{\prime}\left(c_{t}\right) M_{t}^{-1}$ is a martingale with respect to the shifted, hedgeable filtration $\left(\mathscr{H}_{t+1}\right)$.

The fact that the quotient $e^{-\rho t} u^{\prime}\left(c_{t}\right) M_{t}^{-1}$ is a martingale is one of the most remarkable properties of incomplete markets in the class $\mathfrak{C}$. We note that nothing similar can arise for general incomplete markets.

Definition 5.3 Let $\mathbf{C}(\mathbf{w}, \mathbf{M})=\left(c_{t}\right)$ be the optimal consumption stream satisfying the budget constraints (5.2) and the first order conditions of Proposition 5.2. We denote by

$$
W_{t}=M_{t}^{-1} E\left[\sum_{\tau=t}^{T} c_{\tau} M_{\tau} \mid \mathscr{G}_{t}\right]
$$

the intertemporal wealth at time $t$.

Note that the budget constraints (5.2) can be written down in the following equivalent form:

$$
W_{t}-E\left[W_{t} \mid \mathscr{H}_{t}\right]=\mathrm{Y}_{t}-E\left[\mathrm{Y}_{t} \mid \mathscr{H}_{t}\right]=\mathrm{I}(\mathbf{w}, t)
$$

That is, the unhedgeable component of the intertemporal wealth coincides with the unhedgeable component of the intertemporal value of endowment.

\subsection{Precautionary savings}

Consider a simple incomplete market model with only one period risk free bonds available for trading and suppose, for the simplicity, that the risk free rate $r$ is constant. Then, Proposition 5.2 means that the marginal utility process $u^{\prime}\left(c_{t}\right), t \geqslant 0$ is a martingale with respect to $\mathscr{G}$. This result is well known in macroeconomic theory (see, e.g., [21, Chap. 16]). In particular, this implies that at infinite horizon the consumption must grow to infinity. Proposition 5.2 is a direct extension of this claim to the whole class $\mathfrak{C}$ of incomplete markets. This is the precautionary savings effect. 
We will need a

Definition 5.4 Let $g(x): \mathbb{R}^{+} \rightarrow R^{+}$be the inverse of the marginal utility function $u^{\prime}(x)$, that is $g\left(u^{\prime}(x)\right)=x$ for all $x \in \mathbb{R}^{+}$.

We first consider the case of purely idiosyncratically incomplete markets (see, Sect. 4.1). In this case, idiosyncratic income shocks are the only source of uncertainty. Consequently, if the endowment process $\mathbf{w}$ is adapted to the aggregate filtration $\mathscr{F}$, there are no idiosyncratic shocks, the market becomes complete and the optimal consumption stream satisfies the first order conditions

$$
e^{-\rho t} u^{\prime}\left(c_{t}\right)=u^{\prime}\left(c_{0}\right) M_{t}
$$

That is,

$$
c_{t}=g\left(u^{\prime}\left(c_{0}\right) e^{\rho t} M_{t}\right)
$$

Similarly,

$$
c_{t}=g\left(u^{\prime}\left(c_{t-1}\right) e^{\rho} M_{t} M_{t-1}^{-1}\right)
$$

for all $t \geqslant 1$. Idiosyncratic shocks together with the convexity of marginal utility generate precautionary savings, as we show in

Proposition 5.5 Let $\mathbf{c m}=\left(c_{t}, t=0, \ldots, T\right)$ be the optimal consumption satisfying the Euler equations of Proposition 5.2. Suppose that $u^{\prime \prime \prime}(x)>0$, that is the agent has preferences for skewness. Then,

$$
\mathrm{P}_{\mathscr{H}}^{t} c_{t} \geqslant g\left(u^{\prime}\left(c_{t-1}\right) e^{\rho} M_{t} M_{t-1}^{-1}\right)
$$

If $u^{\prime \prime \prime}(x)<0$, then the inequalities above hold in the opposite direction.

Proposition 5.5 means that, given the consumption at time $t-1$, an agent exposed to idiosyncratic risk will consume more in the next period than an agent with no idiosyncratic component in the individual endowment. Thus, the size of precautionary savings depends on the curvature of the marginal utility. It is a well known result in macroeconomics (see, e.g., $[19,27,28,32])$ in the case when only one period risk free bonds are available for trading. To the best of our knowledge, almost nothing is known about precautionary savings in the presence of risky assets. Proposition 5.5 shows that all the conventional economic intuition directly extends to the whole class $\mathfrak{C}$ of incomplete markets. Furthermore, we conjecture, that such percautionary savings effects arise only in incomplete markets from the class $\mathfrak{C}$.

In the case of a scale invariant CRRA utility the precautionary savings effect is even stronger. Note that, for

$$
u(x)=\frac{x^{1-\gamma}-1}{1-\gamma}
$$

the optimal consumption stream in a complete market satisfies

$$
c_{t}=e^{-\rho \gamma^{-1}} M_{t}^{-\gamma^{-1}} c_{0}
$$

We have

Proposition 5.6 Let $\mathbf{C}=\left(c_{t}, t=0, \ldots, T\right)$ be the optimal consumption stream for a CRRA utility with risk aversion $\gamma$. Then,

$$
\frac{c_{t}}{e^{-\rho t \gamma^{-1}} M_{t}^{-\gamma^{-1}}}
$$


is a submartingale with respect to $\left(\mathscr{H}_{t+1}, t=0, \ldots, T\right)$. That is,

$$
\frac{E\left[c_{t} \mid \mathscr{H}_{t}\right]}{c_{t-1}} \geqslant \frac{e^{-\rho t \gamma^{-1}} M_{t}^{-\gamma^{-1}}}{e^{-\rho(t-1) \gamma^{-1}} M_{t-1}^{-\gamma^{-1}}}
$$

Proposition 5.6 shows that for CRRA utilities, in the presence of idiosyncratic risk, the growth

$$
\frac{E\left[c_{t} \mid \mathscr{H}_{t}\right]}{c_{t-1}}
$$

of consumption at each moment of time is larger than the corresponding consumption growth in a complete market. This is a very strong form of precautionary savings that arises because of the scale invariance of CRRA utilities.

\subsection{Recursive structure}

In Sect. 2.2 we have presented a way to construct the optimal consumption stream recursively. The main difference between general incomplete markets and the markets in class $\mathfrak{C}$ is the non-local character of the projections $\mathrm{P}_{\mathscr{L}}^{t}$. By contrast, a conditional expectation acts locally and allows us to solve all the equations state by state. The main ingredient of the recursive construction in Proposition 2.13 is the inversion of nonlinear, non-local monotone maps, described in Lemma 2.11. Inversion of such a map is a difficult problem, that is very nontrivial to implement numerically. By contrast, when $\mathrm{P}_{\mathscr{L}}^{t}=\mathrm{P}_{\mathscr{H}}^{t}$ is a conditional expectation, the corresponding map is local and the problem reduces to inverting (random) functions of one variable, which is a much easier problem. We describe this construction in detail below.

Definition 5.7 Denote by $\mathscr{P}$ the set of all positive endowment processes $\mathbf{w}$, adapted to $\mathscr{G}$.

Fix a subalgebra $\mathfrak{A}$ of $\mathscr{B}$. An $\mathscr{A}$ measurable lower threshold $a(s, \mathbf{w})$ is a map from $\Omega \times \mathscr{P}$ to $(0,+\infty)$, that is $\mathfrak{A}$ measurable for each fixed $\mathbf{w}$. That is, for each fixed $\mathbf{w}$, this is just a $\mathfrak{A}$-measurable random variable.

An $\mathfrak{A}$ measurable random function supported by the lower threshold $a(s, \mathbf{w})$ maps a point $(s, \mathbf{w}) \in \Omega \times \mathscr{P}(\mathscr{G}, \mathbf{M})$ to a real valued function $g(s, \mathbf{w})(x)$ that belongs to $C^{\infty}(a(s, \mathbf{w}),+\infty)$, such that for each fixed $\mathbf{w} \in \mathscr{P}$ and fixed $x \in \mathbb{R}$ the random variable $g(s, \mathbf{w})(x)$ is $\mathfrak{A}$ measurable.

That is, for each fixed $\mathbf{w}$ and fixed state $s, g(s, \mathbf{w})(x)$ is a smooth function, defined on the half line $(a(s, \mathbf{w}),+\infty)$.

Definition 5.8 We denote by esssup $[X \mid \mathfrak{A}]$ conditional, essential supremum of a random variable $X$ on $\Omega$ with respect to the sigma subalgebra $\mathfrak{A} \subset \mathscr{B}$. That is, $\operatorname{esssup}[X \mid \mathfrak{A}]$ is the minimal $\mathfrak{A}$-measurable random variable $Y$, satisfying $Y \geqslant X{ }^{2}$

We will now inductively construct two random functions $F_{t}$ and $G_{t}$ for each $t=1, \ldots, T$. We will need the following technical

Lemma 5.9 Fix a subalgebra $\mathfrak{A}$ of $\mathscr{B}$. Let $\mu(s, \mathbf{w})(x)$ be a $\mathfrak{A}$ measurable random function supported by the lower threshold $a(s, \mathbf{w})$. Suppose that for almost every $s \in \Omega$ and

2 Conditional, essential supremum is easily constructed when $\Omega$ is finite: the algebra $\mathfrak{A}$ is generated by "states", i.e., pairwise disjoint subsets of $\Omega$, such that any set in $\mathfrak{A}$ is a union of a finite number of "states". Then, for each "state", conditional essential supremum is just the maximum over the corresponding subset of $\Omega$. 
every $\mathbf{w} \in \mathscr{P}$, the random function $\mu$ is strictly monotone decreasing on $(a(s, \mathbf{w}),+\infty)$. For convenience, set

$$
A(s, \mathbf{w}):=\operatorname{esssup}[a(s, \mathbf{w}) \mid \mathfrak{A}]
$$

If

$$
\begin{gathered}
\lim _{x \downarrow A} E[\mu(s, x) \mid \mathfrak{A}]=+\infty \\
\lim _{x \rightarrow+\infty} \mu(s, x)=0
\end{gathered}
$$

almost surely, then the unique $\mathfrak{A}$ measurable solution $g(s, \mathbf{w})(x)$ to

$$
E[\mu(s, \mathbf{w})(g(x)) \mid \mathfrak{A}]=x
$$

is an $\mathfrak{A}$ measurable random function supported by the lower threshold 0 . Furthermore, for each $\mathbf{w}$ and almost every $s$, the function $g(s, \mathbf{w})(x)$ is monotone decreasing on $(0,+\infty)$.

Similarly, suppose that $\mu$ is strictly monotone increasing on $(a,+\infty)$ for almost every $s \in \Omega$ and every $\mathbf{w} \in \mathscr{P}$. If

$$
\begin{aligned}
\lim _{x \downarrow a} \mu(s, x) & =b(s, \mathbf{w}) \\
\lim _{x \rightarrow+\infty} \mu(s, x) & =+\infty
\end{aligned}
$$

almost surely, then the unique solution $g(s, \mathbf{w})(x)$ to

$$
E[\mu(s, \mathbf{w})(g(x)) \mid \mathfrak{A}]=x
$$

is an $\mathfrak{A}$ measurable random function supported by the lower threshold

$$
E[b(s, \mathbf{w}) \mid \mathfrak{A}]
$$

Furthermore, for each $\mathbf{w}$ and almost every s the function $g(s, \mathbf{w})(x)$ is monotone increasing on

$$
(E[b(s, \mathbf{w}) \mid \mathfrak{A}],+\infty)
$$

Recall Definition 2.8. Inada conditions imply that the random function

$$
u^{\prime}\left(\mathrm{I}_{T}(\mathbf{w}, \mathbf{M})+x\right)=: \mu(s, \mathbf{w})(x)
$$

supported by the lower threshold

$$
a_{T}(s, \mathbf{w})=-\min \left\{\mathrm{I}_{T}, 0\right\}
$$

satisfies condition (5.6) of Lemma 5.9.

For each endowment process $\mathbf{w}$, set

$$
G_{T}(s, \mathbf{w})(x):=\left(\mathrm{I}_{T}(\mathbf{w}, \mathbf{M})+x\right)
$$

By construction, it is a $\mathscr{G}_{T}$ measurable random function supported by the lower threshold

$$
a_{T}(s, \mathbf{w})=-\min \left\{\mathrm{I}_{T}, 0\right\}
$$

Lemma 5.9 and the conclusion of the preceding paragraph imply that there exists an $\mathscr{H}_{T}$ measurable random function $F_{T}(s, \mathbf{w})(x)$ supported by the lower threshold $a=0$ that is the unique solution to the equation

$$
e^{-\rho} E\left[u^{\prime}\left(G_{T}(s, \mathbf{w})\left(F_{T}(x)\right)\right) \mid \mathscr{H}_{T}\right]=u^{\prime}(x) M_{T} M_{T-1}^{-1}
$$


Continuing inductively, we obtain

Proposition 5.10 Let $G_{T}$ and $F_{T}$ be the random functions given by (5.8) and (5.9). For each $t=1, \ldots, T-1$ there exists a pair of random functions $G_{t}(s, \mathbf{w})(x)$ and $F_{t}(s, \mathbf{w})(x)$ that are respectively $\mathscr{G}_{t}$ and $\mathscr{H}_{t}$ measurable and respectively supported by the lower thresholds $a=0$ and

$$
a_{t}(s, \mathbf{w}):=\operatorname{esssup}\left[\left(E\left[F_{t+1}(s, \mathbf{w})(0) \mid \mathscr{G}_{t}\right]-\mathrm{I}_{t}\right) \mid \mathscr{G}_{t}\right]
$$

They are inductively determined as the unique solutions (recall, Definition 2.8) to the equations

$$
G_{t}(x)+E\left[\frac{M_{t+1}}{M_{t}} F_{t+1}(s, \mathbf{w})\left(G_{t}(x)\right) \mid \mathscr{G}_{t}\right]=x+\mathrm{I}_{t}
$$

and

$$
e^{-\rho} E\left[u^{\prime}\left(G_{t}(s, \mathbf{w})\left(F_{t}(x)\right)\right) \mid \mathscr{H}_{t}\right]=u^{\prime}(x) M_{t} M_{t-1}^{-1}
$$

For almost every $s \in \Omega$ and every nonnegative $\mathbf{w}$ the random functions $F_{t}$ and $G_{t}$, $t=1, \ldots, T$ are monotone increasing in $x$ to the right of their lower thresholds.

Remark 5.11 The random function $F_{t}$ is monotone increasing on $(0,+\infty)$ and consequently has a limit as $x \downarrow 0$. One can show that

$$
F_{t}(s, \mathbf{w})(0):=\lim _{x \downarrow 0} F_{t}(s, \mathbf{w})(x)=\operatorname{esssup}\left[a_{t}(s, \mathbf{w}) \mid \mathscr{H}_{t}\right]
$$

Remark 5.12 Clearly, finiteness of $\Omega$ implies that the random function $u^{\prime}\left(G_{t}(x)\right)$ satisfies condition (5.6) of Lemma 5.9. That is,

$$
\lim _{x \rightarrow A_{t}} E\left[u^{\prime}\left(G_{t}(x)\right) \mid \mathscr{H}_{t}\right]=+\infty
$$

with

$$
A_{t}=\operatorname{esssup}\left[a_{t} \mid \mathscr{H}_{t}\right]
$$

This observation makes it possible to carry out the inductive application of Lemma 5.9, required for the proof of Proposition 5.10.

Recall that

$$
V_{t}(\mathbf{w}, \mathbf{M}):=\mathrm{P}_{\mathscr{H}}^{t}\left[M_{t}^{-1} \sum_{\tau=t}^{T} c_{\tau}(\mathbf{w}, \mathbf{M}) M_{\tau}\right]
$$

We now make the inductive structure of the optimal consumption stream explicit in

Theorem 5.13 Let $\mathbf{C}(\mathbf{w}, \mathbf{M})=\left(c_{t}, t=1, \ldots, T\right)$ be the optimal consumption stream. The consumption $c_{0}(\mathbf{w}, \mathbf{M})$ at time zero is determined by

$$
c_{0}+E\left[M_{1} F_{1}(s, \mathbf{w})\left(c_{0}\right)\right]=E\left[\sum_{t=0}^{T} w_{t} M_{t}\right]
$$

For every $s \in \Omega$, and all $t=1, \ldots, T$,

$$
V_{t}=F_{t}(s, \mathbf{w})\left(c_{t-1}\right)
$$


and

$$
c_{t}=G_{t}(s, \mathbf{w})\left(V_{t}\right)
$$

Here, $F_{t}, G_{t}, t=1, \ldots, T$, are the random functions constructed in Proposition 5.10.

Note that the budget constraints can be rewritten in the form

$$
V_{t}=W_{t}-\mathrm{I}_{t}(\mathbf{w}, \mathbf{M})
$$

By Definition 2.8, $\mathrm{I}_{t}$ is a linear function of the income stream. Consequently,

$$
W_{t}=F_{t}(s, \mathbf{w})\left(c_{t-1}\right)+\mathrm{I}_{t}(\mathbf{w}, \mathbf{M})
$$

(see Definition 5.3) and

$$
c_{t}=G_{t}(s, \mathbf{w})\left(W_{t}-\mathrm{I}_{t}(\mathbf{w}, \mathbf{M})\right)
$$

Note that the construction of the random functions $F_{t}$ and $G_{t}$ can be easily and effectively implemented on a computer because on each step we should solve a one dimensional equation. An application of some version of the Newton procedure will very quickly yield the required result. Thus, our explicit, recursive construction can be easily implemented on the computer.

We would like again to emphasize the difference between the structure of the optimal consumption streams for general incomplete markets (see, Theorem 2.9) and the streams in the markets from $\mathfrak{C}$. In Theorem 2.9, the map $F_{t}$ is not monotone in any sense. Thus, future wealth and thus future consumption are not monotone functions of past wealth. This is a very surprising phenomenon: it might happen that increasing the wealth today will force the agent's consumption and wealth to decrease in some future states. Monotonicity of $F_{t}$ and $G_{t}$, proved in Proposition 5.10, implies that nothing like this can happen for markets in $\mathfrak{C}$. Namely, the following is true:

Theorem 5.14 For markets in the class $\mathfrak{C}$, future consumption and future wealth are monotone increasing functions of past consumption and past wealth.

\subsection{Diminishing marginal propensity to consume}

The functions $F_{t}$ and $G_{t}$ contain all the important economic information about the structure of the optimal consumption stream. For example, the derivative

$$
\frac{\partial c_{t}}{\partial W_{t}}=\frac{\partial G_{t}(s, \mathbf{w})\left(W_{t}-\mathrm{I}_{t}(\mathbf{w}, \mathbf{M})\right)}{\partial W_{t}}
$$

of the consumption with respect to wealth is referred to as the marginal propensity to consume. The next theorem describes important convexity/concavity properties of the functions $F_{t}$ and $G_{t}$.

Theorem 5.15 Let $g(x)$ be the inverse of the function $u^{\prime}(x)$. Suppose that the function $u^{\prime}$ (and then, of course, $g(x))$ is convex, that is, $u^{\prime \prime \prime}(x)>0$ and the function

$$
-\frac{g^{\prime}(x)}{g^{\prime \prime}(x)}
$$

is concave. Then, for every $t=1, \ldots, T$ and almost every $s \in \Omega$ the random function $G_{t}(s, \mathbf{w})(x)$ is jointly concave in the pair $(x, \mathbf{w})$, while the random function $F_{t}(s, \mathbf{w})(x)$ is jointly convex in the pair $(x, \mathbf{w})$. Here, $F_{t}$ and $G_{t}$ are the random functions, constructed 
in Proposition 5.10. If $-\frac{g^{\prime}(x)}{g^{\prime \prime}(x)}$ is not concave, then none of the functions $F_{t}\left(G_{t}\right)$ is convex (concave).

Remark 5.16 Direct calculation shows that conditions of Theorem 5.15 hold true for any HARA utility function with $u^{\prime \prime \prime}>0$. In particular, they hold for all for CRRA and CARA utilities.

An important consequence of Theorems 5.15 and 5.13 is

Proposition 5.17 Under the hypothesis of Theorem 5.15. For each moment of time t,

- Consumption $c_{t}$ is a jointly concave function of wealth $W_{t}$ and endowment process $\mathbf{w}$.

- Future wealth $W_{t+1}$ is a jointly convex function of consumption $c_{t}$ and endowment process $\mathbf{w}$.

Proposition 5.17 has very important economic consequences. The fact that consumption is a concave function of wealth means that the marginal propensity to consume is diminishing. That is, the reacher an agent is, the smaller is the part of the wealth he consumes. Keynes [16] emphasized the importance of the concavity of the consumption function. This fact has been rigorously proved by Carroll and Kimball [2] in the case when only one period risk free bonds are available for trading. Proposition 5.17 establishes a much stronger result. Namely, it shows that the concavity holds for a the whole class $\mathfrak{C}$ of incomplete markets, and that consumption is in fact jointly concave in wealth and endowment. It would be interesting to make statistical investigations of real consumption data analyzing the joint concavity property.

Note that the wealth $W_{t}$ can be decomposed

$$
W_{t}=c_{t}+E\left[\frac{M_{t+1}}{M_{t}} W_{t+1} \mid \mathscr{G}_{t}\right]
$$

into consumption $c_{t}$ at time $t$ and savings

$$
S_{t}=E\left[\frac{M_{t+1}}{M_{t}} W_{t+1} \mid \mathscr{G}_{t}\right]
$$

The last formula reflects the fact that savings $S_{t}$ at time $t$ equal the discounted value of future wealth $W_{t+1}$. Thus, clearly, if consumption $c_{t}\left(W_{t}\right)$ is concave in $W_{t}$, the wealth $W_{t}=W_{t}\left(c_{t}\right)$ is a convex function of consumption. Consequently, the savings

$$
E\left[\frac{M_{t+1}}{M_{t}} W_{t+1} \mid \mathscr{G}_{t}\right]=S_{t}=W_{t}\left(c_{t}\right)-c_{t}
$$

are also a convex function of $c_{t}$. Proposition 5.17 establishes much stronger result: future wealth $W_{t+1}$ is pointwise convex in past consumption.

We will also show how concavity directly relates to precautionary savings. Suppose that the market is idiosyncratically incomplete and let us add an idiosyncratic component $\lambda \mathbf{w}^{\mathrm{I}}$ to the endowment process $\mathbf{w}^{A}$ of an agent. We assume that idiosyncratic shocks have mean zero, conditioned on the aggregate events in $\mathscr{F}$. That is, $\mathbf{P}_{\mathscr{F}} \mathbf{w}^{\mathrm{I}}=0$. Parameter $\lambda$ measures the strength of idiosyncratic risk. Because the shocks have mean zero, the wealth of the agent does not change, but the consumption stream will change because of precautionary savings. The following result is a direct consequence of Proposition 5.17.

Corollary 5.18 Under the hypothesis of Theorem 5.15, let

$$
\mathbf{w}(\lambda)=\mathbf{w}^{A}+\lambda \mathbf{w}^{I}
$$


be the endowment process of the agent with $\mathbf{P}_{\mathscr{F}} \mathbf{w}^{\mathrm{I}}=0$ and $\lambda>0$. Then, consumption $c_{0}(\mathbf{w}(\lambda))$ at time zero is monotone decreasing in $\lambda$, and consequently, the savings $S_{1}$ are monotone increasing in $\lambda$.

Corollary 5.18 establishes a very strong form of precautionary savings: savings are monotone increasing in the size of idiosyncratic risk for the class of utility functions described in Theorem 5.15.

Obviously, all the remarkable structure, arising for markets in $\mathfrak{C}$ completely evaporates when $\mathrm{P}_{\mathscr{L}}$ is not positivity preserving. For example, precautionary savings occur for utilities with $u^{\prime \prime \prime}>0$ because of the Jensen inequality

$$
E\left[u^{\prime}(c) \mid \mathscr{H}_{t}\right]>u^{\prime}\left(E\left[c \mid \mathscr{H}_{t}\right]\right)
$$

Clearly, no inequality of the form

$$
\mathrm{P}_{\mathscr{L}}^{t} u^{\prime}(c)>u^{\prime}\left(\mathrm{P}_{\mathscr{L}}^{t} c\right)
$$

can hold when $\mathrm{P}_{\mathscr{L}}^{t}$ is not positivity preserving. First, a non positivity preserving projection $\mathrm{P}_{\mathscr{L}}^{t}$ will immediately destroy all convexity properties of $u^{\prime}$. Second, if $\mathrm{P}_{\mathscr{L}}^{t} c$ is not positive, $u^{\prime}\left(\mathrm{P}_{\mathscr{L}}^{t} c\right)$ is not even defined.

Similarly, it is clear from the proof of Theorem 5.15 that, without positivity of $\mathrm{P}_{\mathscr{L}}^{t} u^{\prime}$, all the convexity/concavity properties also disappear.

\section{Constant relative risk aversion. Weak idiosyncratic risk}

In this section we obtain an approximate expression for the optimal consumption stream in the special case of purely idiosyncratically incomplete market (see Sect. 4.1) when idiosyncratic risk is small and the utility function is CRRA, $u(x)=\left(x^{1-\gamma}-1\right) /(1-\gamma)$.

We will also use the notations

$$
\begin{gathered}
\mathbf{P}_{\mathscr{H}}=\bigoplus_{t=1}^{T} \mathrm{P}_{\mathscr{H}}^{t} \\
\mathbf{P}_{\mathscr{F}}=\bigoplus_{t=1}^{T} \mathrm{P}_{\mathscr{F}}^{t}
\end{gathered}
$$

Everywhere in this section the market is assumed to be purely idiosyncratically incomplete.

Definition 6.1 Let

$$
\Lambda_{t}=\Lambda_{t}(\rho, \gamma, \mathbf{M}):=M_{t}^{-1} \mathrm{P}_{\mathscr{F}}^{t} \sum_{\tau=t}^{T} e^{-\rho t b} M_{\tau}^{1-b} c_{0}=M_{t}^{-1} \mathrm{P}_{\mathscr{F}}^{t} \sum_{\tau=t}^{T} \mathrm{~cm}_{\tau} M_{\tau}
$$

where

$$
\mathrm{cm}_{t}=e^{-\rho t b} M_{t}^{-b} c_{0}
$$

is the complete market consumption stream and $b=\gamma^{-1}$. Let also (see Definition 2.8)

$$
\mathrm{Y}_{t}(\mathbf{w}, \mathbf{M})=M_{t}^{-1} E\left[\sum_{\tau=t}^{T} w_{\tau} M_{\tau} \mid \mathscr{G}_{t}\right]
$$


and

$$
\mathrm{I}_{t}(\mathbf{w}, \mathbf{M})=\mathrm{Y}_{t}-E\left[\mathrm{Y}_{t} \mid \mathscr{H}_{t}\right]
$$

We define

$$
V_{\tau}^{\mathrm{I}}=\frac{\operatorname{Var}_{\mathscr{F}_{\tau}}\left(\mathrm{I}_{\tau}\right)}{\left(\Lambda_{\tau}\right)^{2}}=\mathrm{P}_{\mathscr{F}}^{\tau} \frac{\operatorname{Var}_{\mathscr{H}_{\tau}}\left(\mathrm{I}_{\tau}\right)}{\left(\Lambda_{\tau}\right)^{2}}
$$

We can now compute the second order Taylor expansion for the optimal consumption stream when idiosyncratic risk is weak. Namely, we consider an agent with an $\mathscr{F}$-adapted endowment stream $\mathbf{w}^{\mathrm{A}}$ and we want to see what happens when we add a small idiosyncratic component $\varepsilon\left(I-\mathbf{P}_{\mathscr{F}}\right) \mathbf{w}^{\mathrm{I}}$, to the endowment. Here, $\varepsilon$ is a small parameter and $\left(I-\mathbf{P}_{\mathscr{F}}\right)$ guarantees that the conditional mean of idiosyncratic shocks is zero.

Theorem 6.2 Let $\mathbf{C}(\varepsilon):=\mathbf{C}(\mathbf{w}(\varepsilon))$ be the optimal consumption stream of the agent with discount rate $\rho$, CRRA utility function $u(x)=\left(x^{1-\gamma}-1\right) /(1-\gamma)$ and endowment process $\mathbf{w}(\varepsilon)=\mathbf{w}^{\mathrm{A}}+\varepsilon\left(I-\mathbf{P}_{\mathscr{F}}\right) \mathbf{w}^{\mathrm{I}}$ with $\mathbf{w}^{\mathrm{A}} \in \mathbf{P}_{\mathscr{F}} H$. Then,

$$
\mathbf{P}_{\mathscr{F}} \mathbf{C}(\varepsilon)=\mathbf{c m}\left(1+\varepsilon^{2} \frac{1}{2}(1+\gamma) \sum_{\tau=1}^{t} V_{\tau}^{\mathrm{I}}+O\left(\varepsilon^{3}\right)\right)
$$

Here, $\mathbf{c m}=\left(\mathrm{cm}_{t}, t=1, \ldots, T\right)$ is the complete market optimal consumption stream. Consequently, the growth rate

$$
\log \frac{\mathrm{P}_{\mathscr{F}}^{t} c_{t}(\varepsilon)}{\mathrm{P}_{\mathscr{F}}^{t-1} c_{t-1}(\varepsilon)}
$$

of the optimal consumption stream is monotone increasing in $\varepsilon$ for all sufficiently small $\varepsilon$ and for any $t=1, \ldots, T$.

Remark 6.3 Recall (see Definition 2.8) that $\mathrm{I}_{t}$ is the unhedgeable part of agent's future idiosyncratic risk and $\Lambda_{\tau}$ (see Definition 6.1) is the value at time $t$ of the future optimal consumption stream in a complete market. Therefore, we can view

$$
\frac{\operatorname{Var}_{\mathscr{F}_{\tau}}\left(\mathrm{I}_{\tau}\right)}{\left(\Lambda_{\tau}\right)^{2}}
$$

as size of future unhedgeable idiosyncratic risk relative to the complete market consumption. Thus, the quantity arising in the expansion (6.1) is the cumulative size of future idiosyncratic risk over the period $[1, \ldots t]$.

Remark 6.4 The second order coefficient in the expansion (6.1) is always nonnegative and strictly positive for generic idiosyncratic risk processes, not belonging to $\mathbf{P}_{\mathscr{F}} H$. This means that the growth rate of the optimal consumption process is monotone increasing in the strength of idiosyncratic risk for sufficiently small $\varepsilon$. As we show in Corollary 5.18 , the expected value of future consumption stream is monotone increasing in $\varepsilon$ for all $\varepsilon$ (not only for sufficiently small). Simple examples show that the monotonicity result of Theorem 6.2 does not hold for strong idiosyncratic risk (large $\varepsilon$ ). The "average" monotonicity of Corollary 5.18 is the best result we can get for strong idiosyncratic risk. 


\section{Appendix A: The derivation of budget constraints and first order conditions}

Lemma A.1 For any two $\mathscr{G}_{t+1}$-measurable random variables $X, Y$ we have

$$
E\left[\mathrm{P}_{\mathscr{L}}^{t+1}(X) Y \mid \mathscr{G}_{t}\right]=E\left[\mathrm{P}_{\mathscr{L}}^{t+1}(Y) X \mid \mathscr{G}_{t}\right]
$$

Proof It suffices to show that for any $\mathscr{G}_{t}$-measurable random variable $Z$,

$$
E\left[\mathrm{P}_{\mathscr{L}}^{t+1}(X) Y Z\right]=E\left[\mathrm{P}_{\mathscr{L}}^{t+1}(Y) X Z\right]
$$

The last claim follows from the self-adjointness of $\mathrm{P}_{\mathscr{L}}^{t+1}$ and the identity $\mathrm{P}_{\mathscr{L}}^{t+1}(Y Z)=$ $Z \mathrm{P}_{\mathscr{L}}^{t+1}(Y)$.

Proof of Lemmas 2.5 and 2.7 By Lemma A.1,

$$
E\left[\left(d_{j, t+1}+p_{j, t+1}\right) R_{t+1} R_{t}^{-1} \mid \mathscr{G}_{t}\right]=E\left[\left(d_{j, t+1}+p_{j, t+1}\right) \mathrm{P}_{\mathscr{L}}^{t+1}\left(R_{t+1} R_{t}^{-1}\right) \mid \mathscr{G}_{t}\right]
$$

That is, $\mathbf{R}$ is a state price density process if and only if $\mathrm{P}_{\mathscr{L}}^{t+1}\left(R_{t+1} R_{t}^{-1}\right)$ is a stochastic discount factor for each $t$.

Let $\mathbf{R}$ be any positive state price density process. Then,

$$
M_{t}=\prod_{\tau=0}^{t} \mathrm{P}_{\mathscr{L}}^{\tau}\left(R_{\tau} R_{\tau-1}^{-1}\right)
$$

is an aggregate SPD process. It remains to show that it is unique. But, any SPD process $\mathbf{M}$ satisfies

$$
E\left[\left(d_{j, t+1}+p_{j, t+1}\right) X M_{t+1} M_{t}^{-1}\right]=E\left[p_{j t} X\right]
$$

for any $\mathscr{G}_{t}$-measurable $X$. Since such variables span $\mathscr{L}_{t+1}$, a variable $M_{t+1} \in \mathscr{L}_{t+1}$ is uniquely determined by these identities.

The budget constraints of Lemma 2.7 follow directly from the following property: a consumption stream $c_{t}=w_{t}+D_{\mathbf{x}, t}$ generated by a portfolio strategy $\mathbf{x}$ satisfies

$$
\mathrm{P}_{\mathscr{G}}^{t} \sum_{\tau=t}^{T}\left(c_{\tau}-w_{\tau}\right) \frac{M_{\tau}}{M_{t}}=D_{\mathbf{x}, t}=\sum_{j=1}^{N}\left(D_{j t}+q_{j t}\right) x_{j t-1} \in \mathscr{L}_{t}
$$

\section{Appendix B: Positivity of the projections}

Proof of Theorem 3.2 Let $1, \ldots, S$ be the states of the sigma algebra $\mathscr{G}_{t}$ (nodes of the event tree at time $t$ ) and $\left\{\delta_{1}, \ldots, \delta_{S}\right\}$ be the standard basis $L_{2}\left(\mathscr{G}_{t}\right)$ with $\delta_{i}\left(s_{i}\right)=1$ and $\delta_{i}\left(s_{j}\right)=0$ for all $j \neq i$.

Suppose that $\mathrm{P}_{\mathscr{L}}^{t}$ is positivity preserving. By construction, $\mathrm{P}_{\mathscr{L}}^{t} 1=1$ and therefore, in the above standard basis, $\mathrm{P}_{\mathscr{L}}^{t}$ is a stochastic matrix with nonnegative entries and the ergodic theorem (see, e.g., [10,21]) implies that

$$
\lim _{r \rightarrow \infty} \frac{1}{r} \sum_{\tau=1}^{r}\left(\mathrm{P}_{\mathscr{L}}^{t}\right)^{\tau}
$$


exists and is a direct some of one dimensional projections. But,

$$
\left(\mathrm{P}_{\mathscr{L}}^{t}\right)^{\tau}=\mathrm{P}_{\mathscr{L}}^{t}
$$

for all $\tau \geqslant 1$ and thus $\mathrm{P}_{\mathscr{L}}^{t}$ is itself a direct sum of one dimensional projections. Since $\mathrm{P}_{\mathscr{L}}^{t} 1=1$ the vectors on which it projects must be characteristic functions of some subsets of $\Omega$. This subsets generate a sigma algebra $\mathscr{H}_{t}$ and, clearly, $\mathrm{P}_{\mathscr{L}}^{t}$ is the conditional expectation with respect to $\mathscr{H}_{t}$.

\section{Appendix C: Convexity and concavity}

We will need two auxiliary lemmas. The first one is well known.

Lemma C.1 Let $H$ be a convex subset of a linear space and $F: H \times \mathbb{R}_{+} \rightarrow \mathbb{R}$,

$$
(h, x) \rightarrow F(h, x)
$$

be jointly concave in $(h, x)$ and strictly monotone in $x$. Let $g(h): H \rightarrow \mathbb{R}_{+}$be the unique solution to the equation

$$
F(h, g(h))=0
$$

Then, if $F$ is monotone decreasing in $x$ then $g$ is convex, and if $F$ is monotone increasing in $x$ then $g$ is concave.

Lemma C.2 Let $\Omega$ be a probability space and $H:=L_{\infty}^{+}$be the set of positive essentially bounded random variables with essentially bounded inverse:

$$
L_{\infty}^{+}:=\left\{X \in L_{\infty}(\Omega) \mid X>0 \text { and } X^{-1} \in L_{\infty}\right\}
$$

Let also $f(x): \mathbb{R}^{+} \rightarrow \mathbb{R}^{+}$be a monotone decreasing function and $g(x)$ its inverse,

$$
g(f(x))=f(g(x))=x, \quad x \in \mathbb{R}
$$

If the function $f(x)$ (and hence $g(x))$ is convex and the function

$$
-\frac{g^{\prime}(x)}{g^{\prime \prime}(x)}: \mathbb{R}^{+} \rightarrow \mathbb{R}^{+}
$$

is concave, then the function $F: H \rightarrow \mathbb{R}^{+}$defined by

$$
F(X)=g(E[f(X)])
$$

is concave.

Proof By direct computation, the Hessian $D^{2} F$ of the function $F$ is given by

$$
\left\langle D^{2} F(X) Y, Y\right\rangle=g^{\prime \prime}(E[Z])\left(E\left[f^{\prime}(X) Y\right]\right)^{2}+g^{\prime}(E[Z]) E\left[f^{\prime \prime}(X) Y^{2}\right]
$$

Let $f^{\prime \prime}(X)$ denote also the operator of multiplication by the function $f^{\prime \prime}(X)$. Then,

$$
D^{2} F(X)=g^{\prime}(E[Z])\left(f^{\prime \prime}(X)\right)^{1 / 2}\left(I+\frac{g^{\prime \prime}(E[Z])}{g^{\prime}(E[Z])} E\left[\frac{\left(f^{\prime}(X)\right)^{2}}{f^{\prime \prime}(X)}\right] \mathbf{P}\right)\left(f^{\prime \prime}(X)\right)^{1 / 2}
$$

where

$$
\mathbf{P} Y=\left(E\left[\frac{\left(f^{\prime}(X)\right)^{2}}{f^{\prime \prime}(X)}\right]\right)^{-1} E\left[\frac{f^{\prime}(X)}{\left(f^{\prime \prime}(X)\right)^{1 / 2}} Y\right] \frac{f^{\prime}(X)}{\left(f^{\prime \prime}(X)\right)^{1 / 2}}
$$


is the orthogonal projection onto the vector $\frac{f^{\prime}(X)}{\left(f^{\prime \prime}(X)\right)^{1 / 2}} \in L_{\infty}^{+}$. Therefore, the Hessian is nonpositive definite if and only if

$$
\frac{g^{\prime \prime}(E[Z])}{-g^{\prime}(E[Z])} E\left[\frac{\left(f^{\prime}(X)\right)^{2}}{f^{\prime \prime}(X)}\right] \leqslant 1
$$

Since $Z=f(X)$ and $g$ is the inverse of $f$, we have $X=g(Z)$. Furthermore, differentiating the identity $g(f(x))=x$ to times, we get that

$$
\frac{\left(f^{\prime}(g(z))\right)^{2}}{f^{\prime \prime}(g(z))}=-\frac{g^{\prime}(z)}{g^{\prime \prime}(z)}
$$

Therefore, inequality (C.2) can be rewritten as

$$
E\left[\frac{g^{\prime}(Z)}{g^{\prime \prime}(Z)}\right] \geqslant \frac{g^{\prime}}{g^{\prime \prime}}(E[Z])
$$

This is simply Jensen's inequality for the function $g^{\prime} / g^{\prime \prime}$.

Proof of Theorem 5.15 We now prove the convexity/concavity property. We prove the claim inductively. If the rewrite the defining equation for $F_{t}$ in the form

$$
g\left(E\left[u^{\prime}\left(G_{t}(s, \mathbf{w})\left(F_{t}\right)\right) \mid \mathscr{H}_{t}\right]\right)-g\left(e^{\rho} u^{\prime}\left(c_{t-1}\right) M_{t-1}^{-1} M_{t}\right)=0
$$

then we are in a position to apply Lemmas C.1 and C.2. Since, by construction, $G_{T}$ is linear and hence concave, and a superposition of two monotone increasing concave functions is again concave, we get the required result. If we know that $F_{t+1}$ is convex, then Lemma C.1 applies to the equation

$$
G_{t} M_{t}+\mathrm{P}_{\mathscr{G}}^{t} F_{t+1}\left(G_{t}\right)-\mathrm{I}_{t}-V_{t}=0
$$

are we get the required concavity of $G_{t}$. The proof is completed by induction.

\section{Appendix D: A toy calculation: the inductive construction of optimal consumption streams in a two period model}

To make the inductive construction of optimal consumption streams immediately accessible, we carry out the procedure in the simplest possible case of a two period model with a toy probability space.

D.1 A toy probability space, sigma algebras and projections

- $\Omega=\left\{s_{1}, \ldots, s_{8}\right\}$ is the probability space with eight elements of equal probability $1 / 8$.

- $\mathscr{G}_{0}=\{\emptyset, \Omega\}$ is the trivial algebra, $\mathscr{G}_{1}$ is the algebra, generated by

$$
\left\{s_{1}, s_{2}, s_{3}, s_{4}\right\}, \quad\left\{s_{5}, s_{6}, s_{7}, s_{8}\right\}
$$

and $\mathscr{G}_{2}=2^{\Omega}$.

- $\mathscr{F}_{0}=\mathscr{F}_{1}=\{\varnothing, \Omega\}$ are trivial algebras and $\mathscr{F}_{2}$ is the algebra, generated by

$$
\left\{s_{1}, s_{2}, s_{5}, s_{6}\right\}, \quad\left\{s_{3}, s_{4}, s_{7}, s_{8}\right\}
$$

- $\mathscr{H}_{0}=\mathscr{H}_{1}=\{\emptyset, \Omega\}$ are trivial algebras and $\mathscr{H}_{2}$ is generated by the sets

$$
\left\{s_{1}, s_{2}\right\}, \quad\left\{s_{3}, s_{4}\right\}, \quad\left\{s_{5}, s_{6}\right\}, \quad\left\{s_{7}, s_{8}\right\}
$$


Lemma D.1 Assumption 2 holds. That is, for $t=0,1$ and any $\mathscr{F}_{t+1}$ measurable random variable $Y, \mathrm{P}_{\mathscr{F}}^{t} Y=\mathrm{P}_{\mathscr{G}}^{t} Y$.

Proof Assumption 2 obviously holds for $t=0$ because $\mathscr{F}_{0}=\mathscr{G}_{0}$ are trivial. For $t=1$, we have to show that for any $\mathscr{F}_{2}$ measurable variable $Y \mathrm{P}_{\mathscr{F}}^{1} Y=\mathrm{P}_{\mathscr{G}}^{1} Y$. Since $Y$ is $\mathscr{F}_{2}$-measurable, it is constant on the sets (D.2). Let $Y_{i}:=Y\left(s_{i}\right)$. Then,

$$
\mathrm{P}_{\mathscr{F}}^{1} Y=E[Y]=\frac{1}{2}\left(Y_{1}+Y_{3}\right)
$$

Conditioning on the sets (D.1), generating $\mathscr{G}_{1}$, and using the fact that $\operatorname{Prob}\left[s_{i}\right]=1 / 8$ for all $i=1, \ldots, 8$, we obtain

$$
E\left[Y \mid\left\{s_{1}, s_{2}, s_{3}, s_{4}\right\}\right]=\frac{1}{2}\left(Y_{1}+Y_{3}\right)=\frac{1}{2}\left(Y_{5}+Y_{7}\right)=E\left[Y \mid\left\{s_{5}, s_{6}, s_{7}, s_{8}\right\}\right]
$$

D.2 Budget constraints and first order conditions

Fix aggregate state price densities $\mathbf{M}=\left(M_{0}, M_{1}, M_{2}\right)$. As usual, $M_{0}=1$. Let $\mathbf{w}=\left(w_{0}\right.$, $w_{1}, w_{2}$ ) be the $\mathscr{G}$ adapted endowment process of the agent. Let $\mathrm{I}_{t}, t=1,2$, be the (random) values of idiosyncratic risk at time $t$, introduced in Definition 2.8. By definition,

$$
\mathrm{I}_{2}(\mathbf{w}, \mathbf{M})=\left(I-\mathrm{P}_{\mathscr{H}}^{2}\right) w_{2} M_{2}=w_{2} M_{2}-\mathrm{P}_{\mathscr{H}}^{2} w_{2} M_{2}
$$

and

$$
\mathrm{I}_{1}(\mathbf{w}, \mathbf{M})=\left(\mathrm{P}_{\mathscr{G}}^{1}-\mathrm{P}_{\mathscr{H}}^{1}\right)\left(w_{1} M_{1}+w_{2} M_{2}\right)
$$

Observe that

$$
\begin{aligned}
\mathrm{P}_{\mathscr{H}}^{1} \mathrm{I}_{1} & =0 \\
\mathrm{P}_{\mathscr{H}}^{2} \mathrm{I}_{2} & =0
\end{aligned}
$$

Let for the simplicity $u(x)=\left(x^{1-\gamma}-1\right) /(1-\gamma)$ be a CRRA utility function. Let $\mathbf{C}=$ $\left(c_{0}, c_{1}, c_{2}\right)$ be the optimal consumption stream, satisfying the budget constraints (5.1), (5.2) and the first order conditions (5.2), that in the present case reduce to

$$
\begin{aligned}
& c_{2} M_{2}=V_{2}+\mathrm{I}_{2} \\
& e^{-\rho} \mathrm{P}_{\mathscr{H}}^{2} c_{2}^{-\gamma} M_{2}^{-1}=c_{1}^{-\gamma} M_{1}^{-1} \\
& c_{1} M_{1}+\mathrm{P}_{\mathscr{G}}^{1} c_{2} M_{2}=V_{1}+\mathrm{I}_{1} \\
& e^{-\rho} \mathrm{P}_{\mathscr{H}}^{1} c_{1}^{-\gamma} M_{1}^{-1}=c_{0}^{-\gamma}
\end{aligned}
$$

where,

$$
\begin{aligned}
V_{2} & =\mathrm{P}_{\mathscr{H}}^{2} c_{2} M_{2} \\
V_{1} & =\mathrm{P}_{\mathscr{H}}^{1}\left(c_{1} M_{1}+c_{2} M_{2}\right)
\end{aligned}
$$

Observe that both $M_{1}$ and $V_{1}$ are constants, because $\mathscr{F}_{1}=\mathscr{H}_{1}$ are trivial algebras. This is a peculiarity of the present toy calculation. We use this observation below to simplify some of the equations, but it is not strictly necessary. 
For any random variable $X$ on $\Omega$, we use the abbreviated notation $X\left(s_{i}\right):=X(i)$. The identity (D.5) takes the form

$$
\mathrm{I}_{2}(1)+\mathrm{I}_{2}(2)=\mathrm{I}_{2}(3)+\mathrm{I}_{2}(4)=\mathrm{I}_{2}(5)+\mathrm{I}_{2}(6)=\mathrm{I}_{2}(7)+\mathrm{I}_{2}(8)=0
$$

since all states have the same probability. Similarly, $I_{1}$ is $\mathscr{G}_{1}$ measurable and is therefore constant on the sets (D.1) and takes only two values,

$$
\begin{aligned}
& \mathrm{I}_{1}(1)=\mathrm{I}_{1}(2)=\mathrm{I}_{1}(3)=\mathrm{I}_{1}(4) \\
& \mathrm{I}_{1}(5)=\mathrm{I}_{1}(6)=\mathrm{I}_{1}(7)=\mathrm{I}_{1}(8)
\end{aligned}
$$

Therefore, (D.4) becomes

$$
\mathrm{I}_{1}(1)+\mathrm{I}_{1}(5)=0
$$

Moreover, $V_{2}=\mathrm{P}_{\mathscr{H}}^{2} c_{2} M_{2}$ is constant on the sets (D.3) since it is a $\mathscr{H}_{2}$ measurable random variable. Substituting (D.6) into (D.7), we obtain the equation

$$
e^{-\rho} \mathrm{P}_{\mathscr{H}}^{2}\left(V_{2}+\mathrm{I}_{2}\right)^{-\gamma}=c_{1}^{-\gamma} M_{1}^{-1} M_{2}^{1-\gamma}
$$

for $V_{2}$.

D.3 Two period inductive construction

First observe that (D.12) is equivalent to

$$
e^{-\rho}\left(\left(V_{2}(i)+\mathrm{I}_{2}(i)\right)^{-\gamma}+\left(V_{2}(i)-\mathrm{I}_{2}(i)\right)^{-\gamma}\right)=2\left(c_{1}(i)\right)^{-\gamma} M_{1}^{-1}\left(M_{2}(i)\right)^{1-\gamma}
$$

for $i=1, \ldots, 8$, by combining (D.10) and Prob $\left[s_{i} \mid\left\{s_{i}, s_{i+1}\right\}\right]=1 / 2$. For each fixed $i=1, \ldots, 8$, and all $x>0$, there is a unique solution $y=F_{2}\left(\mathbf{w}, s_{i}\right)(x)$ to the equation

$$
e^{-\rho}\left(\left(y+\mathrm{I}_{2}(i)\right)^{-\gamma}+\left(y-\mathrm{I}_{2}(i)\right)^{-\gamma}\right)=2 x^{-\gamma} M_{1}^{-1}\left(M_{2}(i)\right)^{1-\gamma}
$$

where $F_{2}\left(\mathbf{w}, s_{i}\right)(x)$ is a monotone increasing function of $x>0$. Clearly, $F_{2}\left(\mathbf{w}, s_{i}\right)(x)$ is an $\mathscr{H}_{2}$ measurable random function, supported by the lower threshold 0 because $\mathrm{I}_{2}$ and $M_{2}$ are both $\mathscr{H}_{2}$ measurable. It follows that

$$
V_{2}(i)=F_{2}\left(\mathbf{w}, s_{i}\right)\left(c_{1}(i)\right)
$$

In the special case $\gamma=1$, we mention that

$$
F_{2}\left(\mathbf{w}, s_{i}\right)(x)=\frac{x M_{2}(i) e^{-\rho}+\left(\left(x M_{2}(i) e^{-\rho}\right)^{2}+4\left(\mathrm{I}_{2}(i)\right)^{2}\right)^{1 / 2}}{2}
$$

The next step in the induction is to construct the random function $G_{1}\left(\mathbf{w}, s_{i}\right)(x)$. For this purpose, observe that, by (D.13), the second budget constraint (D.8) becomes

$$
c_{1}(i) M_{1}+\mathrm{P}_{\mathscr{G}}^{1} F_{2}\left(\mathbf{w}, s_{i}\right)\left(c_{1}(i)\right)=V_{1}+\mathrm{I}_{1}(i)
$$

for $i=1, \ldots, 8$. The random variable $c_{1}$ actually takes only two values, since $\mathscr{G}_{1}$ is generated by the two sets (D.1). Therefore, (D.15) is equivalent to ${ }^{3}$

$$
\begin{aligned}
& c_{1}(1) M_{1}+\frac{1}{2}\left(F_{2}\left(\mathbf{w}, s_{1}\right)\left(c_{1}(1)\right)+F_{2}\left(\mathbf{w}, s_{3}\right)\left(c_{1}(1)\right)\right)=V_{1}+I_{1}(1) \\
& c_{1}(5) M_{1}+\frac{1}{2}\left(F_{2}\left(\mathbf{w}, s_{5}\right)\left(c_{1}(5)\right)+F_{2}\left(\mathbf{w}, s_{7}\right)\left(c_{1}(5)\right)\right)=V_{1}+I_{1}(5)
\end{aligned}
$$

\footnotetext{
3 Note that, by (D.11), $I_{1}(5)=-I_{1}(1)$. We do not use this fact explicitly.
} 
For all

$$
x>a(1):=\frac{1}{2}\left(\left|\mathrm{I}_{2}(1)\right|+\left|\mathrm{I}_{2}(3)\right|\right)-\mathrm{I}_{1}(1)
$$

there is a unique solution $y=G_{1}\left(\mathbf{w}, s_{1}\right)(x)$ to the equation

$$
y M_{1}+\frac{1}{2}\left(F_{2}\left(\mathbf{w}, s_{1}\right)(y)+F_{2}\left(\mathbf{w}, s_{3}\right)(y)\right)=x+I_{1}(1)
$$

Similarly, for all

$$
x>a(5):=\frac{1}{2}\left(\left|\mathrm{I}_{2}(5)\right|+\left|\mathrm{I}_{2}(7)\right|\right)-\mathrm{I}_{1}(5)
$$

there is a unique solution $y=G_{1}\left(\mathbf{w}, s_{5}\right)(x)$ to the equation

$$
y M_{1}+\frac{1}{2}\left(F_{2}\left(\mathbf{w}, s_{5}\right)(y)+F_{2}\left(\mathbf{w}, s_{7}\right)(y)\right)=x+I_{1}(5)
$$

By direct examination, both functions on the right hand sides of (D.18), (D.20) are monotone increasing in $y$ and therefore (D.18), (D.20) can be rewritten as

$$
c_{1}(i)=G_{1}\left(\mathbf{w}, s_{i}\right)\left(V_{1}\right)
$$

for $i=1,5$. The random function $G_{1}$ is monotone increasing and $\mathscr{G}_{1}$ measurable with $\mathscr{G}_{1}$ measurable lower threshold $a$ taking values $a(1), a(5)$ on the sets (D.1) (see (D.17) and (D.19)).

The last step is to determine the function $V_{1}=F_{1}\left(c_{0}\right)$. It is determined by combining (D.9) and (D.21) into

$$
\left(\left(G_{1}\left(\mathbf{w}, s_{1}\right)\left(V_{1}\right)\right)^{-\gamma}+\left(G_{1}\left(\mathbf{w}, s_{5}\right)\left(V_{1}\right)\right)^{-\gamma}\right)=2 c_{0}^{-\gamma} M_{1}
$$

\section{Appendix E: Weak idiosyncratic risk}

The action of the Jacobian

$$
D(\mathbf{C})=\frac{\partial \mathbf{C}(\mathbf{w}, \mathbf{M})}{\partial \mathbf{w}}
$$

in the case when the idiosyncratic component of $\mathbf{w}$ vanishes (that is, $\mathbf{w}=\mathbf{w}^{\mathrm{A}}=\mathbf{P}_{\mathscr{F}} \mathbf{w}$ ) takes a simple form. Recall that

$$
\Lambda_{t}=\mathrm{P}_{\mathscr{F}}^{t} \sum_{\tau=t}^{T} \mathrm{~cm}_{\tau} M_{\tau}
$$

Let

$$
\mathbf{Q}=\mathbf{P}_{\mathscr{G}}-\mathbf{P}_{\mathscr{H}}
$$

A direct calculation shows that

Lemma E.1 Let $\mathbf{w} \in \mathbf{P}_{\mathscr{F}} H$ and

$$
\mathbf{N}=\operatorname{diag}\left(\Lambda_{t}\right)_{t=1}^{T}
$$

be the multiplication operator by the process $\Lambda_{t}$. Then,

$$
\left.D(\mathbf{C})\right|_{\mathbf{w}}=B:=\mathbf{c m} J \mathbf{N}^{-1} \mathbf{Q} J^{*} \mathbf{M}
$$


Similarly, the following is true:

Lemma E.2 The second derivative

$$
D^{2}(\mathbf{C})=\frac{\partial^{2} \mathbf{C}}{\partial \mathbf{w}^{2}}
$$

satisfies

$$
D^{2}(\mathbf{C})(\mathbf{w}, \mathbf{w})=(1+\gamma)(I-D(\mathbf{C}))\left[\mathbf{C}^{-1}(D(\mathbf{C}) \mathbf{w})^{2}\right]
$$

Note that $\mathbf{P}_{\mathscr{F}} \mathbf{Q}=0$ and therefore, when $\left(I-\mathbf{P}_{\mathscr{F}}\right) \mathbf{w}=0$ (complete market),

$$
\mathbf{P}_{\mathscr{F}} D(\mathbf{C})=\mathbf{P}_{\mathscr{F}} \mathbf{c m} J \mathbf{N}^{-1} \mathbf{Q} J^{*} \mathbf{M}=\mathbf{c m} J \mathbf{N}^{-1} \mathbf{P}_{\mathscr{F}} \mathbf{Q} J^{*} \mathbf{M}=0
$$

Consequently,

$$
\begin{aligned}
\mathbf{P}_{\mathscr{F}} \mathbf{C}(\mathbf{w}) & =\mathbf{c m}+\varepsilon^{2} \frac{1}{2}(1+\gamma) \mathbf{c m}^{-1}(D(\mathbf{C}) \mathbf{w})^{2} \\
& =\mathbf{c m}\left(1+\varepsilon^{2} \frac{1}{2}(1+\gamma) \sum_{\tau=1}^{t} V_{\tau}^{\mathrm{I}}+O\left(\varepsilon^{3}\right)\right)
\end{aligned}
$$

\section{References}

1. Carroll, C.: Buffer stock saving and the life cycle/permanent income hypothesis. Q. J. Econ. CXII(1), $1-56$ (1996)

2. Carroll, C., Kimball, M.: On the concavity of the consumption function. Econometrica 64(4), 981992 (1996)

3. Constantinides, G.M., Duffie, D.: Asset pricing with heterogenous consumers. J. Polit. Econ. 104(2), 219-240 (1996)

4. Cuoco, D.: Optimal consumption and equilibrium prices with portfolio constraints and stochastic income. J. Econ. Theory 72(1), 33-73 (1997)

5. Dreze, J.H., Modigliani, F.: Consumption under uncertainty. J. Econ. Theory 5(3), 308-335 (1972)

6. Duffie, D., Fleming, W., Soner, H.M., Zariphopoulou, T.: Hedging in incomplete markets with HARA utility. J. Econ. Dyn. Control 21, 753-782 (1997)

7. Duffie, D., Huang, C.-F.: Implementing Arrow-Debreu equilibria by continuous trading of few long-lived securities. Econometrica 53(6), 1337-1356 (1985)

8. Duffie, D., Protter, P.: From discrete- to continuous-time finance: weak convergence of the financial gain process. Math. Financ. 2(1), 1-15 (1992)

9. El Karoui, N., Jeanblanc-Picque, M.: Optimization of consumption with labor income. Financ. Stoch. 2, 409-440 (1998)

10. Feller, W.: An Introduction to Probability Theory and Its Applications. Wiley, New York (1966)

11. Goll, T., Kallsen, J.: A complete explicit solution to the log-optimal portfolio problem. Ann. Appl. Probab. 13(2), 774-799 (2003)

12. Gollier, C.: The Economics of Risk and Time. MIT Press, Cambridge (2001)

13. He, H.: Convergence from discrete- to continuous-time contingent claim prices. Rev. Financ. Stud. 3(4), 523-546 (1990)

14. Hendersen, V.: Explicit solutions to an optimal portfolio choice problem with stochastic income. J. Econ. Dyn. Control 29(7), 1237-1266 (2005)

15. Heston, S.: A closed-form solution for options with stochastic volatility with applications to bond and currency options. Rev. Financ. Stud. 6(2), 327-343 (1993)

16. Keynes, J.M.: General Theory of Employment, Interest and Money. Cambridge University Press, Cambridge (1936)

17. Kimball, M.S.: Precautionary saving in the small and in the large. Econometrica 58(1), 53-73 (1990)

18. Kimball, M.S.: Standard risk aversion. Econometrica 61, 589-611 (1993)

19. Leland, H.E.: Saving and uncertainty: the precautionary demand for saving? Q. J. Econ. 82, 465-473 (1968) 
20. Levhari, D., Srinivasan, T.N.: Optimal saving under uncertainty. Rev. Econ. Stud. 36, 153-163 (1969)

21. Ljungqvist, L., Sargent, T.J.: Recursive Macroeconomic Theory. MIT Press, Cambridge (2000)

22. Lusardi, A.: Permanent income, current income, and consumption: evidence from two panel data sets. J. Bus. Econ. Stat. 14(1), 81-90 (1996)

23. Malamud, S., Trubowitz, E.: Asset Pricing in Idiosyncratically incomplete Markets. Working paper, SSRN (2006). http://ssrn.com/abstract=912788

24. Malamud, S., Trubowitz, E., Wüthrich, M.: Market Consistent Pricing of Insurance Products. Working paper, SSRN (2007). http://ssrn.com/abstract=970549

25. Menezes, C.F., Auten, G.E.: The theory of optimal saving decisions under income risk. Int. Econ. Rev. 19(1), 253-258 (1978)

26. Merton, R.C.: Optimum consumption and portfolio rules in a continuous-time model. J. Econ. Theory 3(4), 373-413 (1971)

27. Miller, B.L.: Optimal consumption with a stochastic income stream. Econometrica 42, 253-266 (1974)

28. Miller, B.L.: The effect on optimal consumption of increased uncertainty in labor income in the multiperiod case. J. Econ. Theory 13, 154-167 (1976)

29. Musiela, M., Zariphopoulou, T.: An example of indifference prices under exponential preferences. Financ. Stoch. 8, 229-239 (2004)

30. Musiela, M., Zariphopoulou, T.: A valuation algorithm for indifference prices in incomplete markets. Financ. Stoch. 8, 399-414 (2004)

31. Rotschild, M., Stiglitz, J.E.: Increasing risk II: Its economic consequences. J. Econ. Theory 3(1), 66-84 (1971)

32. Sandmo, A.: The effect of uncertainty on saving decisions? Rev. Econ. Stud. 37, 353-360 (1970)

33. Schweizer, M.: On the minimal martingale measure and the Fo"llmer-Schweizer decomposition. Stoch. Anal. Appl. 13, 573-599 (1995)

34. Schweizer, M.: A minimality property of the minimal martingale measure. Stat. Probab. Lett. 42, 27-31 (1999)

35. Sibley, D.S.: Permanent and transitory income effects in a model of optimal consumption with wage income uncertainty. J. Econ. Theory 11, 68-82 (1975)

36. Souleles, N.S.: The response of household consumption to income tax refunds. Am. Econ. Rev. 89(4), 947-958 (1999)

37. Willinger, W., Taqqu, M.S.: Toward a convergence theory for continuous stochastic securities market models1. Math. Financ. 1(1), 55-59 (1991)

38. Zeldes, S.: Optimal consumption with stochastic income: deviations from certainty equivalence. Q. J. Econ. 104(2), 275-298 (1989) 